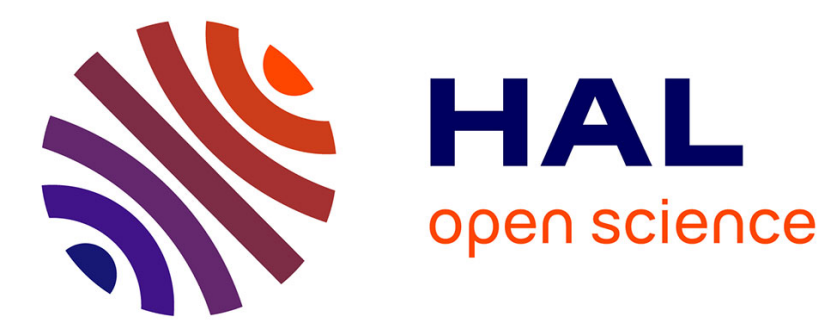

\title{
Le témoignage de Chariton d'Aphrodisias sur la pratique civique des honneurs
}

\author{
Sophie Lalanne
}

\section{To cite this version:}

Sophie Lalanne. Le témoignage de Chariton d'Aphrodisias sur la pratique civique des honneurs. A. Heller et O. van Nijf éd. The politics of Honour in the Greek cities of the Roman Empire, Brill, p. 149-181, 2017. hal-01981728

\section{HAL Id: hal-01981728 \\ https://hal.science/hal-01981728}

Submitted on 15 Jan 2019

HAL is a multi-disciplinary open access archive for the deposit and dissemination of scientific research documents, whether they are published or not. The documents may come from teaching and research institutions in France or abroad, or from public or private research centers.
L'archive ouverte pluridisciplinaire HAL, est destinée au dépôt et à la diffusion de documents scientifiques de niveau recherche, publiés ou non, émanant des établissements d'enseignement et de recherche français ou étrangers, des laboratoires publics ou privés. 


\title{
The Politics of Honour in the Greek Cities of the Roman Empire
}

\author{
Edited by \\ Anna Heller \\ Onno M. van Nijf
}

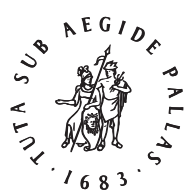

B R I L L

LEIDEN | BOSTON

For use by the Author only | (C 2017 Koninklijke Brill NV 


\section{Contents}

Acknowledgements IX

List of Illustrations $\mathrm{X}$

List of Tables XII

List of Contributors XIII

Introduction: Civic Honours, from Classical to Roman Times 1 Anna Heller and Onno M. van Nijf

PART 1

The Economy of Honour: Financial and Symbolic Exchanges

1 Praise and Honour 31

Olivier Gengler

2 Les timai dans le discours civique et moral à la fin du $\mathrm{I}^{\mathrm{er}}$ siècle ap. J.-C. 59

Anne Gangloff

3 La gestion des statues honorifiques à Rhodes à la fin du $\mathrm{I}^{\mathrm{er}}$ siècle ap. J.-C. d'après le Rhodiakos de Dion de Pruse (Or. XXXI) 81 Henri Fernoux

4 The Financing of Public Honours in Greece during the Roman Imperial Period

The Case of Honorary Statues in the Cities of the Greek Mainland 109 Francesco Camia

\section{PART 2}

Honorific Communities: Competition and Negotiation

5 Le témoignage de Chariton d'Aphrodisias sur la pratique civique des honneurs 149

Sophie Lalanne 
6 Un-civic Benefactions?

Gifts to Non-citizens and Civic Honours in the Greek Cities of the Roman East 182

Arjan Zuiderhoek

7 The Refusal of the Highest Honours by Members of the Urban Elite in Roman Asia Minor 199

Christina T. Kuhn

8 Decrees Awarding Offices for Life and by Hereditary Right as Honours 220

Nikos Giannakopoulos

\section{PART 3}

The Impact of Rome: Integration and Domination

9 Romans in the poleis of Greek Mainland and Adjacent Islands: The Evolution of Their Relations in the Light of Honorific Texts 245 Sofia Zoumbaki

10 Les honneurs des cités d'Asie aux proches des gouverneurs 272 Gabrielle Frija

11 Curateurs de cités et honneurs civiques 291 Éric Guerber

12 Honouring Senators and Equestrians in the Graeco-Roman East 317 Annika B. Kuhn

13 Le premier des citoyens à Pergame sous le Haut-Empire : C. Antius Aulus Iulius Quadratus 339

Olivier Ventroux

PART 4

Cities and Empire: Honours between Local and Global

14 Martyriai: Civic Honours and Imperial Government 373 Christina Kokkinia 
15 On the Rhetoric of Imperial Majesty

Elements of the Ideological Interaction between Emperor and Imperial Society on the Basis of Civic Decrees, Imperial Pronouncements and Literary Testimonies in the Greek East 386

Kostas Buraselis

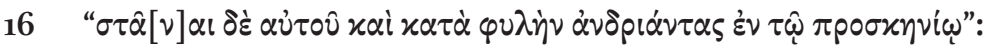

Honorary Statues in the Theatres of Roman Greece 397 Valentina Di Napoli

17 The Agora as Setting for Honorific Statues in Roman Greece $43^{2}$ Christopher Dickenson

18 L'iconographie des honneurs civiques statuaires pour les notables d'époque impériale 455

Martin Szewczyk

19 Le reflet des honneurs 496

Jean-Baptiste Yon

Index of Sources $\quad 527$

A Literary texts 527

B Inscriptions 528

C Papyri 530

D Numismatic Sources 530

Index of Names and Subjects 531 


\title{
Le témoignage de Chariton d'Aphrodisias sur la pratique civique des honneurs
}

\author{
Sophie Lalanne
}

Le roman de Chariton d'Aphrodisias est généralement considéré comme le plus ancien des romans que l'Antiquité nous ait transmis. Il s'agit d'une fiction en prose et en langue grecque, qui met en scène la fille du célèbre général syracusain Hermocrate, Callirhoé, ainsi que son jeune époux Chairéas. Comme les autres romans grecs qui ont été rédigés par la suite, le récit rapporte la séparation des deux jeunes héros, une longue série d'épreuves qui leur permet de manifester et développer les qualités essentielles à leur condition de jeunes gens bien nés, puis leurs retrouvailles et leur retour dans la cité de leurs pères.

Le roman Callirhoé a été composé par un auteur qui se nomme Chariton d'Aphrodisias et qui se dit « avocat du rhéteur Athénagoras » (1.1.1). Selon les plus récentes estimations ${ }^{1}$, il serait possible de dater la composition du roman entre 41 et 61 ap. J.-C., soit entre le début du règne de Claude, après l'assassinat de Caligula par un certain Cassius Chaerea, et la mort du poète satirique Perse qui recommande, dans une satire brocardant les incultes, de leur donner après déjeuner une «Callirhoé » qui serait non pas le nom d'une courtisane, comme on l'a souvent cru à l'époque moderne, mais le titre du roman de Chariton, alors déjà célèbre ${ }^{2}$. Le style du roman a en outre été rapproché de celui d'autres auteurs du I ${ }^{\text {er }}$ siècle ap. J.-C. comme Philon d'Alexandrie, Flavius Josèphe ou Plutarque ${ }^{3}$. L'action quant à elle se déroule, comme nous l'avons indiqué, sous la stratégie d'Hermocrate, le général syracusain qui remporta la victoire en 413 av. J.-C. contre les Athéniens, au terme de la célèbre « expédition de Sicile», plusieurs années donc après cette bataille maintes fois célébrée dans le roman, mais avant 407, date de la mort d'Hermocrate qui laissa une fille derrière lui.

Il faut préciser que, le plus souvent, les romanciers de langue grecque ne prennent pas la peine de décrire une réalité qui leur est familière. Ils plantent

\footnotetext{
1 Tilg 2010, "Chariton's date", 36-79.

2 Sur Cassius Chaerea « adulescens animi ferox », cf. Tacite, Annales, 1.32. Persius, Satire, 1.134, reste relativement énigmatique : «post prandia Callirhoen do. »

3 Ruiz Montero 1991; Hernandez 1994.
} 
leur décor en quelques mots, ne décrivant que ce dont l'action rend la mention absolument nécessaire, ainsi que ce qui contribue à donner au récit une « couleur locale » ou, au contraire, une teinte exotique. Dans les cités de l'Orient romain où tout le monde sait ce qu'est un théâtre, un port, un temple ou une agora, et où les modèles architecturaux ne sont pas d'une infinie variété, où tout le monde sait également très précisément ce qu'est un esclave, un magistrat, un mariage ou un procès, un mot suffit à faire surgir un monde. À l'inverse, lorsque les romanciers livrent au lecteur de longues ekphraseis, comme c'est le cas d'Achille Tatius ou d'Héliodore, dont la participation au phénomène culturel de la Seconde Sophistique ne fait guère de doute, ces descriptions ne portent jamais sur des faits de la vie quotidienne, des pratiques sociales ou des lieux de la vie civique, mais sur des œuvres d'art, des jardins et des animaux rares. Le réalisme des ekphraseis ne s'attache jamais à décrire une réalité ordinaire.

Le roman de Chariton d'Aphrodisias qui, avec celui d'Héliodore, est sans conteste celui qui évoque le plus nettement l'univers de la cité, nous apporte, dans quatre circonstances différentes, un témoignage personnel sur la pratique des honneurs civiques, bien attestée dans les sources épigraphiques de la même époque, en particulier sur les bases inscrites des statues honorifiques qui ornaient les rues et les lieux publics. Notre fil d'Ariane sera donc le relevé analytique des informations délivrées par le roman Callirhoé, en regard du contenu des inscriptions contemporaines ${ }^{4}$. Après avoir passé en revue rapidement les caractères spécifiques de la représentation de la cité dans le roman de Chariton, nous nous pencherons sur les quatre textes dans lesquels le peuple décerne des honneurs pour étudier l'identité et le statut des honorandi, les bienfaits récompensés, les honneurs décernés et la procédure institutionnelle suivie.

\section{La représentation de la cité dans le roman de Chariton d'Aphrodisias}

Pas d'honneurs civiques sans vie civique. Or la cité n'est pas le décor privilégié par les romanciers. L'Orient romain hellénophone, tel qu'il est décrit dans le roman grec, est bien un monde de cités, mais où l'on voyage et où les cités ne sont que des jalons dans le long périple qui mène les héros aux portes de

4 Je tiens ici à remercier les participants du colloque de Tours, en particulier J.-L. Ferrary, H. Pleket et les deux éditeurs de ce volume, A. Heller et O. van Nijf, pour leurs précieuses remarques et suggestions. 
l'oikouménè. Les sanctuaires par exemple sont au moins aussi importants que les cités ${ }^{5}$. Il est donc rare que les héros s'y attardent ${ }^{6}$. La plupart du temps, l'action se déroule dans un entre-deux où sévissent pirates, brigands et rebelles (la mer, la Cilicie, la chôra égyptienne) ou dans des royaumes orientaux (la Babylonie, l'Égypte, l'Éthiopie) où les Grecs exilés, privés de points de repère, sont offerts en pâture aux passions insatiables de despotes orientaux. Lorsqu'ils résident dans des cités, l'action est souvent circonscrite au péristyle ou à la salle de banquet d'une maison de ville, ou bien à la chôra environnant la ville. Des cinq romans complets, le roman Callirhoé, le plus ancien chronologiquement, est celui qui campe le plus nettement un décor à proprement parler civique.

Dans le roman de Callirhoé, plusieurs cités sont évoquées : Syracuse en premier lieu, patrie des deux jeunes héros, Milet, où Callirhoé est vendue comme esclave puis épousée par l'homme le plus important de toute l'Ionie, mais aussi Athènes, Priène et Tyr. En réalité, seule Syracuse présente les caractéristiques institutionnelles de la vie en cité, les autres cités offrant des images plus stéréotypées. Milet est avant tout la capitale de l'Ionie, et en tant que telle la cité où réside Dionysios, le premier des notables ioniens, le second époux de Callirhoé, et où séjournent également les satrapes de Carie et de Lydie - l'Ionie est alors sous domination perse. L'action se déroule essentiellement dans la propriété de campagne de Dionysios, donnant sur le bord de mer et comprenant un sanctuaire d'Aphrodite. On trouve tout de même quelques mentions de la polis, ainsi que de la foule des Milésiens qui célèbrent en masse le mariage de Dionysios (3.2.10-17), la naissance de son fils (3.8.3-5) et les funérailles de Chairéas (4.4.7-12), mais rien n'indique alors une intervention officielle du dèmos. Il semble plutôt que la foule participe à des événements de la vie privée de Dionysios auxquels celui-ci a souhaité donner une publicité particulière, et à l'occasion desquels il peut faire preuve de générosité envers ses concitoyens. Athènes, quant à elle, est décrite non sans humour comme une ville procédurière où le peuple est trop bavard, l'Aréopage expéditif et les magistrats plus durs que des tyrans, selon l'image empruntée aux Guêpes d'Aristophane. Priène est une cité de taille moyenne où des étrangers ne peuvent passer inaperçus, et Tyr une forteresse à prendre, allusion évidente à la prise de la ville par Alexandre en 332 av. J.-C.

À Syracuse en revanche, Hermocrate, en tant que stratège, prend les mesures d'urgence qui s'imposent $(3.3 .2-3 ; 3.3 .8 ; 8.6 .2-4)$ ou fait des propositions qu'il soumet à l'Assemblée $(1.5 .7 ; 3.4 .3 ; 3.4 .16-17)$. C'est de toute évidence le

\footnotetext{
5 Sur Delphes, $c f$. Rougemont 1992 et, plus généralement, Lebdiri 2015, 83-120.

6 Sur Athènes dans le roman grec, $c f$. Oudot 1992.
} 
dèmos de Syracuse qui prend les décisions dans un régime s'affichant donc comme démocratique. Apparaissent également des bouleutes et un tribunal ad hoc tiré au sort par les magistrats de la cité. Il s'agit donc d'une configuration politique qui nous autorise à reconnaître la pratique civique des honneurs habituellement décernés aux notables de la cité. S'il n'est pas totalement exclu que ce soit également le cas pour la cité de Milet, nous ne disposons pas pour cette cité d'éléments d'information équivalents. Il ne semble donc pas que Chariton ait souhaité donner de la Milet perse l'image d'une cité autonome où les décisions se prennent de manière collective.

Nous complèterons ce rapide survol en proposant quelques éléments d'analyse de vocabulaire. La première remarque est que le mot timè, qui évoque le plus souvent l'honneur rendu par la cité à un individu méritant, n'est pas fréquent dans le roman grec et n'apparaît jamais sous la forme plurielle qui est précisément celle qui renvoie le plus souvent à la pratique des honneurs civiques ${ }^{7}$. La seconde remarque est que lorsque le mot timè revêt une connotation officielle, il désigne généralement une fonction publique, le plus souvent un sacerdoce (chez Achille Tatius, 8.10.6, et surtout chez Héliodore, 1.19.4; $1.22 .2 ; 1.22 .7 ; 7.5 .4)$. Enfin, un nombre limité de cas évoquent explicitement un honneur rendu par la cité à un individu et c'est, sans trop de surprise, uniquement dans le roman de Chariton d'Aphrodisias et à Syracuse, où la structure de la cité est la plus clairement dessinée.

Évidemment, il y a quelques difficultés à projeter sur la Syracuse contemporaine d'Hermocrate, donc la cité sicilienne de la deuxième moitié du ve siècle av. J.-C., une pratique politique qui s'est développée considérablement aux époques hellénistique et impériale. Cependant, le fait que Chariton évoque à quatre reprises l'usage des honneurs civiques suffit à rendre compte de l'importance qu'il accorde à cette manifestation de la vie civique, au risque de commettre lui-même une forme d'anachronisme. Il n'est donc pas injustifié d'étudier une pratique que Chariton donne comme soubassement à des épisodes décisifs de son récit, tant que nous ne commettons pas l'imprudence de l'attribuer, sous la forme où elle est évoquée, à la période qu'il décrit, mais bien à celle à laquelle il vécut.

7 La plupart du temps, le terme relève du lexique ordinaire et évoque ce qui est honorable, l'estime, la valeur, la récompense (chez Chariton, par exemple, $3.2 .7 ; 4.1 .8 ; 5.6 .1 ; 5.9 .2 ; 7.2 .6$ ). Ainsi, le mot n'apparaît qu'en ce sens chez Longus, et seulement dans sa forme verbale, à deux reprises $(1.31 .2 ; 2.23 .4)$. Des autres significations que le mot timè peut revêtir, le prix de vente d'un esclave est l'une des plus courantes, notamment chez Chariton (1.10.6 ; 1.14.3 ; $1.14 .9 ; 2.5 .11 ; 5.7 .5)$ et Xénophon d'Éphèse $(2.2 .5 ; 2.11 .9 ; 5.7 .9)$. 
Une autre question qui se pose est la nature du régime politique en vigueur à Syracuse à l'époque d'Hermocrate. Le prestigieux général est présenté par Chariton comme le premier des citoyens et le plus éminent des stratèges de Syracuse dans un régime politique où le peuple est seul maître des décisions qui le concernent. L'action se déroule en effet à Syracuse durant la période où la cité fut régie par des institutions démocratiques, entre les règnes tyranniques de Gélon et ses frères et celui de Denys l'Ancien, donc entre 466 et 406 av. J.-C. ${ }^{8}$. C'est bien de la fin de cette époque que l'on date le floruit d'Hermocrate, alors chef du parti oligarchique, vainqueur des Athéniens en 413 av. J.-C. et adversaire du démocrate Dioclès qui pourrait bien être caché derrière le nom d'Ariston, deuxième personnage de la cité et père du héros Chairéas. C'est parce qu'il est décrit comme le principal adversaire politique d'Ariston qu'Hermocrate doit être convaincu par le peuple de laisser sa fille épouser le fils de celui-ci. Dans la réalité, la fille d'Hermocrate, Arétè, épousa Denys l'Ancien peu avant que son père décédât et que son époux s'emparât du pouvoir. On voit que Chariton procède sans trop de scrupules à une sorte de condensé historique qui lui permet de tisser en quelques paragraphes la trame de son « histoire d'amour qui s'est déroulée à Syracuse » (1.1.1). Il ne s'agit donc pas pour lui de valoriser un modèle de cité démocratique puisqu'Hermocrate, à qui il donne la prééminence, est précisément le chef du parti aristocratique, mais plutôt d'évoquer un régime dont les principes institutionnels ont servi d'exemple à la plupart des cités grecques à compter de l'époque hellénistique. De manière générale, le régime politique qui régit la cité de Syracuse selon Chariton n'est pas très éloigné de la démocratie telle qu'elle apparaît à travers les témoignages épigraphiques de l'époque impériale ${ }^{9}$.

\section{Lorsque le peuple de Syracuse décerne des honneurs : les textes disponibles}

Quatre extraits du roman de Chariton décrivent précisément la procédure par laquelle le peuple de Syracuse peut décerner des honneurs à l'un de ses citoyens les plus éminents ou à un étranger particulièrement méritant. Il s'agit

8 Rutter 2000 ; Robinson 2000 et 2011.

9 Le régime politique des cités grecques répond sous l'Empire à une définition du terme assez large : «Est démocratique tout régime qui, ni tyrannique ni aristocratique, comporte une Assemblée ouverte à l'ensemble des citoyens et votant les décisions importantes, élit et contrôle les magistrats, et garantit une justice impartiale protégeant les humbles. » $C f$. Fernoux 2011, 18 et n. 11. 
dans les trois premiers textes d'honneurs rendus directement ou indirectement à Hermocrate et à sa fille, Callirhoé; le quatrième texte expose une série de témoignages de gratitude dont bénéficient successivement son gendre Chairéas, puis le meilleur ami de celui-ci, Polycharme, puis les trois cents Grecs qui ont combattu à leurs côtés contre le roi de Perse et se voient offrir la citoyenneté syracusaine.

Le premier texte (1.1.11-13) se situe au tout début du roman, peu après que Chairéas et Callirhoé se sont rencontrés et sont tombés amoureux l'un de l'autre. Or les deux pères, Hermocrate et Ariston, sont les deux premiers citoyens de Syracuse et s'opposent sur le terrain politique: le peuple se saisit de l'occasion offerte par une réunion légale de l'Assemblée pour demander à Hermocrate d'accorder sa fille en mariage à Chairéas en dépit de cette inimitié. L'honneur fait au prestigieux stratège consiste donc dans la validation officielle et publique du mariage de sa fille Callirhoé, procédure essentielle dans une cérémonie qui repose sur le témoignage des présents. Le fait sera rappelé et souligné à la fin du roman : «En ce qui concerne le début de ton récit, le peuple le

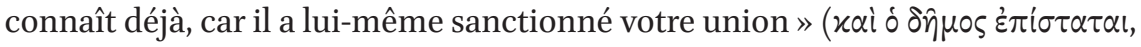

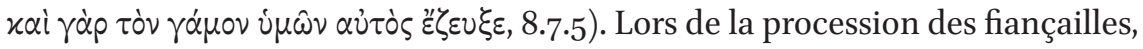
la cité offre le spectacle d'un corps civique uni, comme en rend compte la

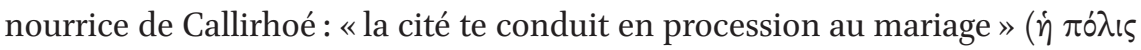
$\sigma \varepsilon \nu \nu \mu \varphi \alpha \gamma \omega \gamma \varepsilon \hat{\imath}, 1.1 .12-13)$. Mais c'est aussi une cité cloisonnée et répartie en différentes catégories d'âge et de sexe, comme c'est le cas généralement dans les

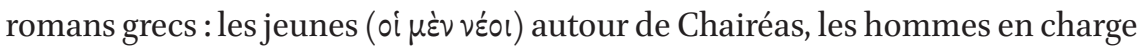

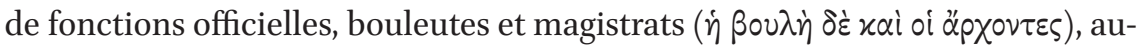

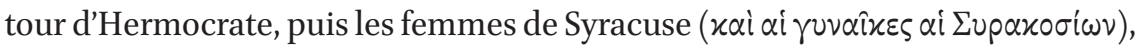
sans nul doute les épouses des citoyens, qui, en dépit de leur exclusion de la citoyenneté et malgré l'absence de Callirhoé, prennent leur place dans cette représentation officielle de la cité.

Le deuxième texte (1.5.2-1.6.5) se situe immédiatement après la mort apparente de Callirhoé suite à un acte de violence de son jeune époux, Chairéas, rendu féroce par la jalousie, et au procès de celui-ci qui demande pour luimême le châtiment le plus sévère. Contre toute attente, Hermocrate demande aux juges d'acquitter son gendre et d'offrir sans plus tarder une sépulture à sa fille. Le peuple obtempère immédiatement et décide d'offrir à la jeune défunte des funérailles publiques. La pompe somptueuse de ces funérailles est pré-

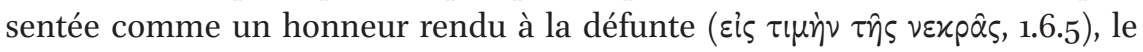
terme timè étant donc ici d'un usage exceptionnel. Le luxe de la procession est le second élément marquant: lit d'or massif, vêtements et étoffes de prix, or et argent de la dot, cadeaux divers, enfin toute la fortune de Chairéas. Les honneurs rendus à un membre de la famille du premier des citoyens sont à 
la hauteur du rang de celui-ci dans la cité. Nous retiendrons surtout que ces

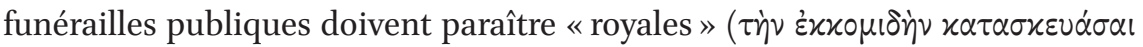

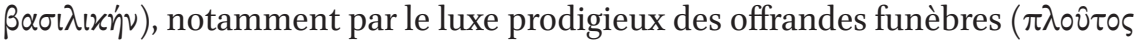

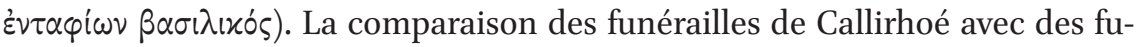
nérailles héroïques, dans le cadre d'une comparaison avec Ariane endormie

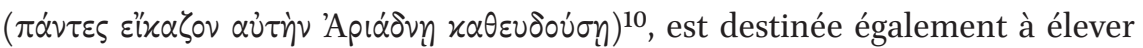
Hermocrate et sa famille au-dessus des simples citoyens. Le troisième élément est le déroulé de la procession civique qui voit défiler dans l'ordre les cavaliers de Syracuse en grande tenue, les hoplites, le Conseil et le peuple entourant Hermocrate, les femmes de citoyens tout de noir vêtues, les offrandes faites à la morte, puis le lit funèbre porté par les éphèbes et la foule des habitants de la cité de Syracuse. On ne sait pas exactement dans quelle catégorie défile Chairéas, mais il semble que ce soit auprès de sa fortune personnelle, car l'expression plaintive de son intention de faire un holocauste de ses biens en l'honneur de son épouse défunte prend la forme d'un discours indirect libre qui suggère une prise de parole accompagnant le cortège.

Dans le troisième texte $(3 \cdot 4 \cdot 3-3 \cdot 5 \cdot 3)$, Hermocrate demande à ses concitoyens d'envoyer une ambassade officielle recouvrer sa fille $(\pi \varepsilon \dot{\varepsilon} \mu \psi \alpha \tau \varepsilon \pi \rho \varepsilon \sigma \beta \varepsilon \dot{c} \alpha \nu$

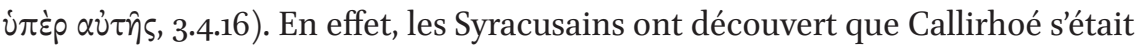
réveillée dans son tombeau et avait été enlevée par des pirates. S'étant spontanément rassemblés en masse au bord de la mer, ils sont invités par Hermocrate à se réunir au théâtre pour mener une enquête officielle sur la disparition du corps de Callirhoé et éventuellement juger les criminels (3.4.3). Effectivement, le chef des pirates Théron, responsable de l'enlèvement de Callirhoé ainsi que de sa vente sur la côte ionienne, est arrêté en mer par Chairéas et assez vite convaincu de culpabilité, sur le témoignage à charge d'un simple pêcheur (3.4.5-16). Le peuple décide alors de partir en masse à la recherche de la jeune fille, tandis que les bouleutes se portent presque tous volontaires (3.4.17). Mais c'est Hermocrate qui définit les modalités selon lesquelles sera composée l'am-

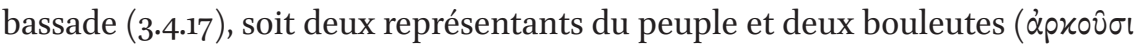

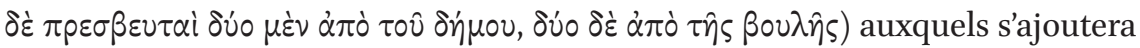
Chairéas, et cela après avoir remercié ses concitoyens pour l'honneur qui lui

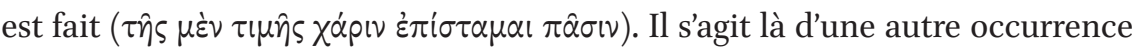
du mot timè au sens d'«honneur» dans le roman de Chariton. La gratification offerte à Hermocrate est alors validée officiellement et l'assemblée levée (3.4.18).

10 Ariane est honorée, à l'époque impériale, comme épouse de Dionysos, et leur couple est souvent décrit comme modèle de l'amour conjugal. Cf. Vatin 2004. 
Dans le quatrième et dernier texte que nous avons retenu (8.7.1-8.8.14),

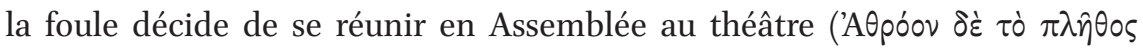

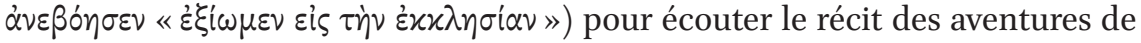
Chairéas et Callirhoé. De même que le peuple a sanctionné l'union de Chairéas et Callirhoé au début du roman, de même il octroie un caractère officiel et cérémoniel à leurs retrouvailles. Chairéas fait alors au peuple qui l'a mandaté le rapport de son ambassade, rapport dans lequel il dresse la liste de ses exploits

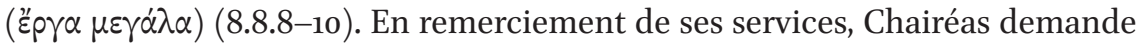
ensuite deux sortes différentes d'honneurs pour ses compagnons d'armes. Pour Polycharme, il demande la validation officielle et publique de son mariage avec sa propre sœur et l'octroi d'une part du butin en guise de dot. Si nous ne connaissons pas d'honneur équivalent dans les sources épigraphiques, nous pouvons néanmoins souligner qu'il fait écho au mariage de Chairéas et Callirhoé eux-mêmes au début du roman. Le dèmos accorde volontiers cette

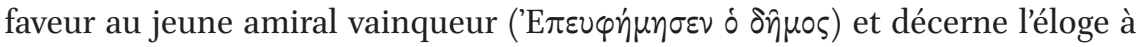
son ami fidèle pour avoir « rendu à la cité des services dignes d'Hermocrate et de Chairéas » (8.8.12-13). Puis Chairéas demande l'octroi collectif de la citoyenneté aux trois cents Grecs qu'il a choisis dans l'armée de l'Égyptien et qui ont combattu à ses côtés - pas un n'a péri, semble-t-il, dans les combats, preuve de leur valeur. Les citoyens s'étant prononcés favorablement, les nouveaux

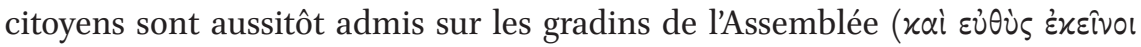

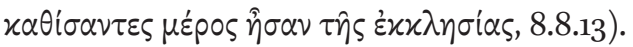

\section{Qui mérite d'être honoré par les Syracusains?}

L'honorandus des premier et troisième textes est Hermocrate. Dans le premier texte, le peuple de Syracuse interpelle directement le premier des stratèges pour le prier de donner son accord au mariage de sa fille, et l'assentiment de celui-ci est analysé par Chariton comme le fruit du patriotisme ( $\varphi$ ìó $\pi \alpha \tau p ı \varsigma$, 1.1.12 $)^{11}$. Le troisième texte met en scène quant à lui l'envoi officiel d'une ambassade de la cité chargée de ramener Callirhoé à Syracuse. Hermocrate évoque explicitement l'honneur qui lui est ainsi rendu et en remercie ses concitoyens

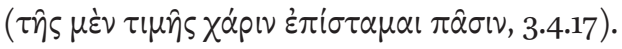

Dans le second texte, en revanche, l'identité de la personne honorée est moins évidente. À la fin du texte, les funérailles somptueuses qui sont offertes à Callirhoé sont décrites comme un honneur rendu à la défunte (

11 Le terme de «philopatris » est lui-même un titre très courant à l'époque impériale ; il est généralement décerné par acclamation. Cf. Robert 1965, 215. 
vexpâs). Cependant, la mention de cette timè est faite incidemment, après la description de la procession, pour annoncer le pillage du tombeau de la jeune femme: «Mais ce qui apparemment était un honneur rendu à la défunte fut le commencement de grands malheurs » (1.6.5). Par ailleurs, rien dans le texte n'évoque ni ne souligne les qualités et les bienfaits que Callirhoé pourrait avoir manifesté envers la cité de Syracuse. Seule sa beauté est évoquée en d'autres occasions, en premier lieu lorsque son père Hermocrate et elle-même sont présentés : «Hermocrate, stratège de Syracuse, le fameux vainqueur des Athéniens, avait une fille, qui s'appelait Callirhoé - une splendeur de jeune

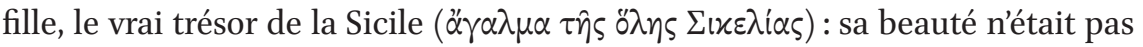
humaine, mais divine $[. .]. »(1.1 .1-2)^{12}$. Certes, sa jeunesse peut expliquer ce silence - Callirhoé est âgée d'une quinzaine d'années - et l'on peut rapprocher sa situation de celle d'Apollonis fille de Proclès, honorée dans un décret de consolation de la cité de Cyzique qui semble devoir être daté du deuxième quart du $\mathrm{I}^{\text {er }}$ siècle ap. J.-C. ${ }^{13}$. Apollonis y est honorée «pour la valeur de ses

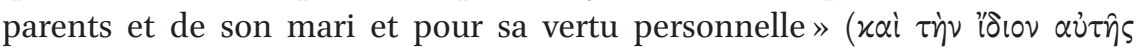

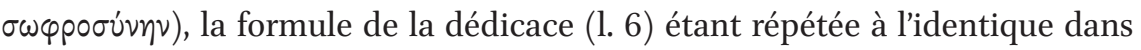
le décret honorifique (l. $5^{6}$ et $87-88$ ), sans autre précision. Si l'on peut relever le fait que la jeune fille est honorée pour une qualité, la chasteté ou la tempérance ( $\sigma \omega \varphi p o \sigma u ́ v \eta)$, qui est une qualité souvent louée chez les jeunes filles et les

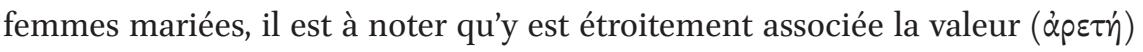
des membres de sa famille : cette valeur familiale est offerte aux regards de la cité et à la considération publique en un ensemble uni et harmonieux, à la manière de ces groupes familiaux dont la statuaire avait pour fonction d'honorer et de conserver le souvenir.

Enfin, la nature même de la procession funèbre, à forte connotation civique et militaire, nous laisse à penser qu'il s'agit aussi bien d'honorer le stratège Hermocrate que sa fille Callirhoé. D'une certaine manière, les honneurs rendus à Hermocrate semblent avoir été étendus à sa fille sans susciter une rhétorique différente de celle qui sied à son père ${ }^{14}$. C'est ce que suggère le fait que

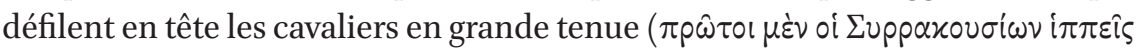

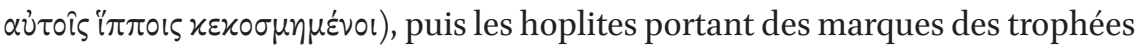

\footnotetext{
12 Les traductions qui suivent sont celles de G. Molinié dans l'édition des Belles Lettres, collection des Universités de France, Paris, 1989. Voir plus récemment Brethes $2016 .$.

13 Sur cette inscription et sur son commentaire, $c f$. Schwertheim 1978; Sève 1979 ; Ehrhardt 2008 ; et l'article de C. Kuhn dans le présent volume.

14 Cf. C. Kuhn dans le présent volume, sur le fait que les femmes honorées ne le sont pas différemment des hommes pour lesquels a été conçue la pratique même des honneurs civiques.
} 


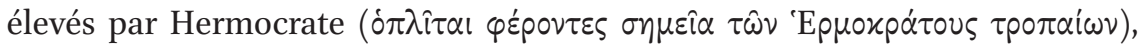
puis le corps civique, entouré des bouleutes, formant la garde personnelle du

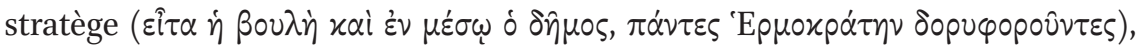

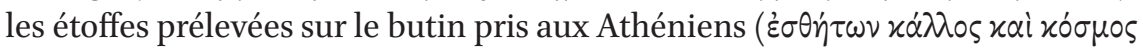

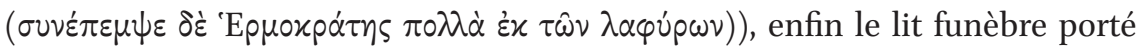

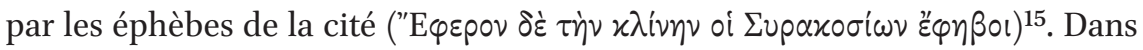
le décret de consolation de Cyzique en l'honneur d'Apollonis, qui peut là aussi nous servir d'élément de comparaison, les corps représentant la cité sont, dans l'ordre, «les prytanes en charge, les magistrats éponymes, les enfants, les éphèbes, les citoyens et tous les hommes libres de la cité, de même que les jeunes filles, les citoyennes et les autres femmes libres habitant la cité » (1. 42-47). Le caractère mélangé de la procession, ouverte aux enfants, aux jeunes filles, aux étrangers et aux étrangères, est assez caractéristique de la pompe funèbre d'une jeune fille à peine entrée dans sa vie de femme mariée. Les éléments civiques n'y sont pas fortement soulignés. Au contraire, Hermocrate et sa fille sont étroitement associés dans un honneur civique qui valorise essentiellement les qualités de stratège du père.

Dans le quatrième texte, ce sont Chairéas, Callirhoé, Polycharme et trois cents mercenaires grecs qui étaient à la solde de l'Égyptien, donc étrangers à la cité de Syracuse, qui sont honorés par la cité assemblée et augmentée du nombre des épouses de citoyens (8.7.1). Le mot timè n'apparaît pas, mais la procédure est assez claire. Chairéas et Callirhoé sont d'abord l'objet d'un éloge public, Chairéas de la part des hommes siégeant au théâtre, Callirhoé de la part des femmes, puis tous deux ensemble de la part de la foule tout entière (8.7.2). Callirhoé est donc honorée une fois de plus aux côtés de son kurios qui n'est plus alors son père mais son époux. Ensuite, Chairéas se voit offrir l'honneur de raconter publiquement ses exploits au théâtre devant toute la cité, tandis que Callirhoé est allée se reposer (8.7.3). En effet, ce récit de Chairéas, qui est encouragé par une longue prise de parole d'Hermocrate et qui prend d'abord la forme d'un rapport d'ambassade, tourne sur la fin, à partir du moment où l'ora-

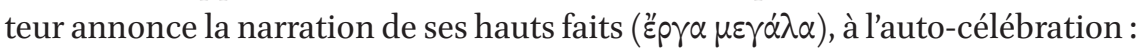
«Tyr était imprenable; je l'ai conquise; nommé amiral, j’ai battu sur la mer le Grand Roi et occupé Arados, où le Roi avait installé à l'écart la reine et tous ces trésors que vous avez vus. J'aurais pu rendre l'Égyptien maître de toute l'Asie, s'il n'avait été tué au combat, en mon absence. Enfin, je vous ai acquis l'amitié du Grand Roi [...]. Pour ma part, j’ai mené ici les plus braves des Grecs et les

15 Les habitués du gymnase jouent un rôle important depuis l'époque hellénistique tardive dans l'organisation des cortèges funéraires offerts en guise d'honneurs posthumes. $C f$. Chankowski 2010, 225. 
volontaires égyptiens. Il viendra d'Ionie une autre flotte qui vous sera amicale : elle sera conduite par le petit-fils d'Hermocrate. » (8.8.8-10.) Ce récit composé de faits d'armes et de succès diplomatiques est rapporté par leur auteur d'une manière si flatteuse et si péremptoire qu'il ne manque pas de faire penser aux Res gestae Divi Augusti par lesquelles Auguste fit lui-même connaître la liste de ses hauts faits (8.8.8-10). Il est en tout cas accueilli par des prières du peuple ( $\varepsilon \dot{x}$ xi) qui marque ainsi son approbation, et immédiatement suivi de la part de Chairéas d'une demande de remerciements publics envers Polycharme. Après avoir été honoré par la cité à la demande d'Hermocrate, Chairéas propose donc au peuple de reporter une part de sa reconnaissance sur son fidèle Patrocle. Polycharme reçoit donc aussi un éloge public et obtient pour gratification supplémentaire d'être marié à la sœur de Chairéas lors de noces publiques. Ces honneurs lui sont décernés pour avoir rendu à la cité des «bienfaits dignes

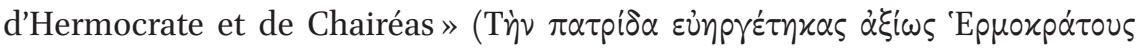
xai Xaıp乏́ou, 8.8.13). Les trois cents mercenaires grecs qui ont combattu à leurs côtés dans la guerre qui a opposé l'Égypte à la Perse obtiennent ensuite collectivement la citoyenneté syracusaine. Aussitôt la proposition adoptée et le décret rédigé (8.8.14), les trois cents hommes sont admis à siéger dans les rangs de l'Assemblée. Aussi bien Polycharme dans la formule ci-dessus ( $\alpha \xi i \omega \varsigma)$

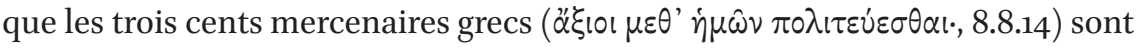
dits axioi, donc dignes de la cité qui les honore. Ces honneurs, qui sont proposés à chaque fois par Chairéas, paraissent aussi comme des honneurs indirects qui sont faits au jeune homme pour récompense de sa victoire navale sur le roi de Perse. Comme l'indique la formule ci-dessus, qui met sur le même plan Hermocrate et son gendre en dépit du jeune âge de ce dernier et de son absence de légitimité à exercer une fonction officielle, il est important pour l'équilibre du roman que Chairéas soit réintroduit dans la cité avec le statut de digne successeur d'Hermocrate. C'est un schéma récurrent du roman grec que de mettre en scène cette passation de pouvoir entre les hommes d'âge mûr (pères, beaux-pères, figures tutélaires) et les jeunes héros ${ }^{16}$.

Il est intéressant de souligner que les bienfaits récompensés dans le roman de Chariton d'Aphrodisias sont exclusivement des exploits militaires, à l'exception éventuellement des funérailles de Callirhoé, si l'on y voit la reconnaissance d'un mérite personnel, et de son éloge final. En effet, les honorandi ne sont jamais loués dans les romans pour leur générosité de bienfaiteurs ou pour l'exercice d'une magistrature, mais uniquement pour leurs faits d'armes. Lorsqu'Hermocrate se voit presser par le peuple d'offrir sa fille en mariage au fils de son adversaire politique, Ariston, il est dit explicitement que ce mariage

16 Lalanne 2006, 118-122, 156-159. 


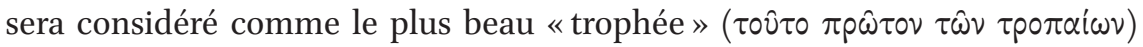
de celui qui est interpellé par le peuple comme «grand stratège » $(\mu \varepsilon \dot{\gamma} \gamma \alpha \varsigma$

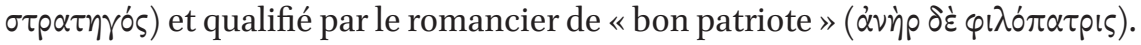
C'est donc en remerciement des exploits guerriers qu'il a accomplis pour la cité de Syracuse lors de sa victoire sur les Athéniens dans la guerre du Péloponnèse que le stratège est honoré par le peuple d'une cérémonie de mariage public pour sa fille unique. La victoire de 413 est en effet évoquée à de multiples reprises dans le roman ${ }^{17}$. Les noces de Chairéas et Callirhoé sont elles-mêmes alors comparées à la célébration d'une victoire militaire (1.1.13). Le deuxième honneur qui est conféré à Hermocrate, à savoir les funérailles publiques de sa fille, n'est pas explicitement rattaché à sa victoire passée, mais le défilé des soldats de Syracuse (cavaliers, hoplites, éphèbes) et le transport des trophées de guerre et des étoffes prélevées sur le butin pris aux Athéniens peuvent le laisser penser.

Dans le troisième texte (3.4.3-3.5.3), c'est en souvenir toujours de ses victoires

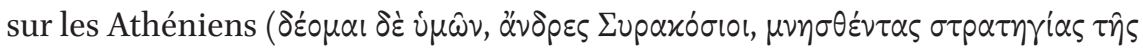

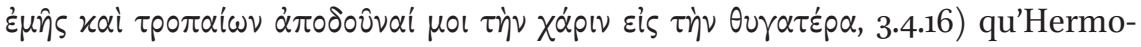
crate demande à ses concitoyens d'envoyer une ambassade officielle recouvrer sa fille $(\pi \varepsilon \dot{\varepsilon} \mu \alpha \tau \varepsilon \pi \rho \varepsilon \sigma \beta \varepsilon \dot{\alpha} \alpha \nu$ i $\pi \dot{\varepsilon} \rho \alpha \dot{v} \tau \hat{\zeta} \varsigma)$. Par ailleurs, le choix de la trière ami-

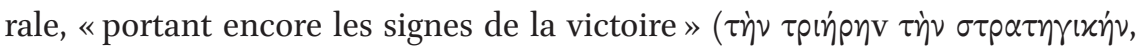

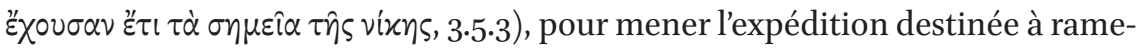
ner Callirhoé, offre l'occasion d'un nouveau rappel des exploits militaires pour lesquels est honoré l'illustre stratège ${ }^{18}$.

Lorsque Chairéas et ses compagnons sont les récipiendaires des honneurs conférés par le peuple de Syracuse, il s'agit toujours de les remercier et de les féliciter pour leur victoire contre le roi de Perse. La gloire de ce combat semble rejaillir sur la cité tout entière, alors même que Chairéas et Polycharme ont combattu, en mercenaires bénévoles, pour l'Égyptien révolté contre la domination perse et que les trois cents Grecs ont été rémunérés pour leurs services. Encore une fois, la comparaison est établie avec la victoire des Syracusains sur les Athéniens en 413 av. J.-C. Fait curieux, la victoire de Chairéas n'est jamais comparée à une bataille qui se serait déroulée au cours des guerres médiques. On peut comprendre que Chariton n'ait pas souhaité célébrer une

$17 C f$. pour les références explicites, 1.1.1, 1.11.2, 2.6.3, 3.10.8, 5.8.8, 6.7.10, 7.2.3, 7.5.8.

18 L'épisode peut évoquer également l'envoi par les Athéniens de la «Salaminienne» qui était chargée de porter les messages officiels, et qui fut envoyée pour arrêter Alcibiade alors qu'il commandait l'expédition de Sicile (Th., 6.41 et 6.53). Autre rappel discret de la célèbre victoire d'Hermocrate... 
bataille navale qui aurait pu rehausser la gloire des Athéniens que le romancier semble peu apprécier par ailleurs, mais pourquoi ne pas évoquer les Thermopyles?

Enfin, l'événement que constituent les retrouvailles de Chairéas et Callirhoé suivi de leur retour à Syracuse, et qui donne lieu à la quatrième manifestation d'octroi d'honneurs civiques, est encore l'occasion de célébrer les exploits militaires du jeune homme. En effet, le dèmos remercie les dieux et leur rend grâce en comparant ce jour à celui de la victoire que Chairéas vient de remporter

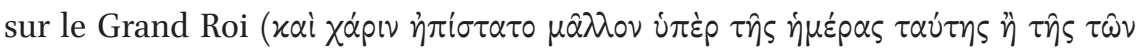
$\varepsilon \dot{\tau} t v(x i \omega v, 8.7 .2)$. Sans bénéficier encore du titre et de la fonction de stratège, Chairéas s'est comporté en amiral et a fait le meilleur usage du commandement militaire que lui avait conféré l'Égyptien : prise de Tyr, victoire navale sur le roi de Perse, prise d'un butin plus considérable que le trésor des Athéniens, acquisition de nouveaux alliés pour Syracuse (les trois cents mercenaires, des volontaires égyptiens et les Ioniens). Comme nous l'avons suggéré, le lustre de sa victoire retombe sur la cité tout entière. De même, Polycharme est récompensé pour des «bienfaits dignes d'Hermocrate et de Chairéas » qui ne peuvent être que le renfort apporté à Chairéas sur le champ de bataille (en plus du soutien psychologique permanent qu'il a apporté à son ami, mais qui ne peut faire l'objet d'une procédure d'octroi d'honneurs civiques), ainsi que les trois cents soldats grecs qui sont gratifiés de la citoyenneté syracusaine et les nombreux soldats égyptiens qui ont suivi Chairéas à Syracuse et qui, sans être admis dans la citoyenneté, sont dotés par lui de lots de terre à cultiver et sont donc installés sur le territoire même de la cité (8.8.14). Est-ce à dire qu'ils travailleront pour son compte sans posséder la terre qu'ils cultiveront, ou bien faut-il comprendre qu'ils sont dotés par Chairéas du droit de posséder la terre de la cité, privilège d'enktèsis que la cité n’a pas validé?

Quoi qu'il en soit, l'exclusivité militaire qui se dégage de cette analyse des honneurs civiques peut s'expliquer par le fait que, tout en souhaitant mettre l'accent sur l'histoire d'amour de Chairéas et Callirhoé, le roman de Chariton réserve à la guerre une place éminente. Elle forme en effet dans tous les romans grecs l'horizon culturel dans lequel doivent évoluer les héros ${ }^{19}$ et, dans le cas de Callirhoé, elle accentue la dimension civique du cadre de l'action. Ajoutons à cela le fait que la guerre se prête certainement mieux au développement d'une intrigue romanesque que les actes d'évergétisme ou que le financement d'une fête, pourtant plus souvent évoqués dans les inscriptions des $\mathrm{I}^{\mathrm{er}}-\mathrm{II}^{\mathrm{e}}$ siècles ap. J.-C.

19 Lalanne 2006, 125-128. Ce statut de la guerre dans le roman grec n'est cependant pas sans ambiguïté, la figure du soldat combattant s'effaçant en partie derrière celle du soldat rentrant dans sa patrie. 


\section{Quels types d'honneurs?}

Nous avons vu que les honneurs pouvaient être décernés de manière individuelle ou collective. Le premier cas est le plus fréquent. Le second est un cas ordinaire d'honneur conféré à un notable auquel l'un des membres les plus proches de sa famille est associé, dans le cas d'Hermocrate sa fille unique, dans le cas de Chairéas son épouse. Relevons ici que ces honneurs adressés aux proches de notables ne sont pas rares à l'époque impériale ${ }^{20}$.

La nature des honneurs est très variée: funérailles publiques, cortège, fête civique, ambassade officielle de la cité embarquée sur une trière sacrée, éloge public, octroi collectif de la citoyenneté, auquel s'ajoute l'honneur romanesque par excellence que sont les noces publiques. Dans tous les cas, il s'agit de donner un caractère public et civique à un événement de la vie privée du premier des notables. C'est par cette publicité que, dans le roman de Chariton, le citoyen honoré est supposé éprouver l'ampleur de la gratitude de ses concitoyens pour son ardeur à servir sa cité. Concernant les noces publiques, nous disposons d'une inscription, datée entre 2 av. J.-C. et 2 ap. J.-C., dans laquelle un notable honoré par sa cité, Kléanax de Kymè, est remercié pour avoir, « célébrant les noces ( $\gamma \alpha \dot{\mu} \mu \mathrm{)})$ de sa fille, [offert] un repas à la population $(\pi \lambda \hat{\alpha} \theta \circ)^{21} \gg$. Il s'agit donc ici aussi pour les citoyens de Kymè de souligner la publicité volontairement donnée par un notable à un acte de sa vie privée. Cependant, dans ce cas précis, la publicité du mariage est accompagnée d'un acte d'évergésie consistant à offrir un banquet, tandis que, dans le roman de Chariton, si l'évergétisme n'est évidemment pas exclu, le caractère public et officiel de la cérémonie est plutôt la marque d'un honneur conféré par la cité au notable qu'est Hermocrate. Doit-on voir là la représentation idéale d'une cité qui, loin d'être redevable à ses élites de leurs actes de générosité, accorde généreusement titres et honneurs?

Les funérailles de Callirhoé offrent un exemple intéressant d'honneur rendu à une toute jeune femme. Comme nous l'avons souligné précédemment, il est frappant de constater que les corps civiques représentés dans la procession sont destinés à représenter la cité avec la dignité qui sied à la fille d'un stratège éminent. Assez différente de la procession décrite dans le décret de consolation de Cyzique en l'honneur de la jeune Apollonis, la procession qui accompagne le corps de Callirhoé suit l'ordre d'une procession religieuse, en tout cas celle des Panathénées telle qu'on peut la voir décrite sur la frise

$20 \quad C f$. l'article de G. Frija dans le présent volume, concernant les familles des gouverneurs.

21 Hodot 1982, 166-167 pour le texte grec et sa traduction. 
ionique du Parthénon ${ }^{22}$. On y retrouve les mêmes corps constitués et le même ordre rituel: hoplites, magistrats, femmes, offrandes, cavaliers. Cependant, les offrandes funéraires remplacent dans le roman de Chariton le bétail destiné au sacrifice et les cavaliers ouvrent la marche au lieu de la fermer; à la fin de la procession défile en effet la foule des Syracusains. Il n'est pas exclu que Chariton ait souhaité rendre hommage à une œuvre d'art bien connue de tous à son époque, mais il n'est pas anodin qu'il fasse une fois de plus référence à la cité des Athéniens, vaincue par Hermocrate.

Deux autres types d'honneurs méritent un commentaire particulier. Tout d'abord, nous évoquerons le magnifique tombeau offert à Callirhoé (1.6.5). Il ne semble pas que celui-ci fasse l'objet d'un traitement particulier de la part de la cité. Les tombeaux étaient généralement situés en un lieu bien choisi sur un terrain appartenant à la famille ou dont la famille faisait alors l'acquisition ${ }^{23}$. À la différence d'Apollonis (l. 49-50) qui obtient de la cité « le droit d'être enterrée dans les tombeaux de famille de son mari qui se trouvent sur le grand port», donc en ville, ce qui est la marque d'un honneur exceptionnel, c'est Hermocrate qui choisit l'emplacement du tombeau de sa fille, tombeau de sa propre famille situé sur un promontoire en bord de mer, visible de loin par tous les Syracusains. Ce type d'emplacement n'est pas un cas particulier ${ }^{24}$. Les concitoyens d'Hermocrate auraient pu préférer un emplacement plus prestigieux, sur le port ou à l'entrée de la ville ${ }^{25}$, mais celui-ci aurait été moins intéressant pour la suite de l'action, fondée sur le pillage du tombeau par des pirates et l'enlèvement de Callirhoé réveillée de son sommeil léthargique. On relèvera surtout le fait que Callirhoé n'est pas enterrée dans le caveau familial de son mari, comme Apollonis, mais de son père, ce qui peut éventuellement s'expliquer par le fait que Chairéas a été la cause de son décès. En revanche, l'exécution du chef des pirates responsable de son enlèvement, Théron, semble inclus dans l'honneur accordé au stratège par le peuple de Syracuse : le pirate est crucifié devant le tombeau de Callirhoé. «Du haut de sa croix, il voyait la

22 Pour d'autres parallèles, $c f$. Rife 1999, 73-78. Aucune procession n'est aussi proche de la description que donne Chariton que celle qu'expose la frise des Panathénées.

23 Rife 1999, 103.

24 Rife 1999, 100, citant les exemples de nombreux sophistes, comme Polémon de Smyrne, qui furent enterrés en bord de mer, ainsi que plusieurs personnages de romans grecs.

25 C'est l'emplacement que choisit pour sa part Dionysios, à Milet, pour le cénotaphe de Chairéas (4.1.5-6). Le tombeau magnifique, que Callirhoé fait construire sur un modèle tout à fait identique au sien à Syracuse, est élevé sur un promontoire qui permet de le voir de loin et fait face en quelque sorte à celui de l'héroïne. Cette fois encore, il s'agit d'une décision émanant d'un particulier mais, nous l'avons vu, c'est toujours le cas chez Chariton en ce qui concerne la cité de Milet.

For use by the Author only | (c) 2017 Koninklijke Brill NV 
mer sur laquelle il avait emporté, telle une captive, la fille d'Hermocrate, la jeune femme que ne purent prendre même les Athéniens. » (3.4.18). La formule, dont le lyrisme n'a d'égal que l'emphase rhétorique, suggère par un raccourci plaisant que le pirate Théron a commis un crime aussi sombre qu'a été éclatante la victoire navale d'Hermocrate et que son châtiment est décidé par le peuple pour en faire l'antithèse des honneurs décernés au stratège.

Arrêtons-nous ensuite sur l'éloge public deChairéas, Callirhoé et Polycharme. Léloge public de Polycharme revêt une forme relativement attendue ${ }^{26}$, bien qu'en partie transposée dans le registre de la relation personnelle: «Polycharme, homme irréprochable, ami fidèle, le dèmos te témoigne sa reconnaissance. Tu as rendu à la cité des bienfaits dignes d'Hermocrate et de

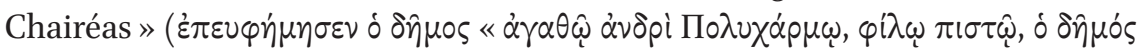

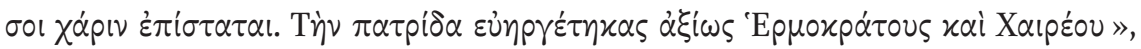
8.8.13). En revanche, l'éloge du couple formé par Chairéas et Callirhoé est plus original, car il s'adresse à chacun des deux héros séparément avant d'associer d'une manière très habituelle la jeune femme à son époux (8.7.2). Le récit public de ses exploits par Chairéas lui-même, qui débute pourtant comme un rapport d'ambassade ${ }^{27}$, se termine en auto-célébration (8.7.3-8.8.11), mais il est demandé par le peuple qui témoigne ensuite de sa satisfaction par l'expression d'un enthousiasme bruyant.

Nous disposons de peu d'informations concernant le financement des honneurs évoqués dans le roman de Chariton. Précisons que les questions économiques et financières n'intéressent nullement les romanciers qui les éludent presque systématiquement. Ainsi, il est impossible de dire à qui incombe la charge financière que représentent les noces de Chairéas et Callirhoé. Qui finance le cortège civique? Qui assume la charge d'organiser la fête somptueuse au cours de laquelle la cité et le plèthos accompagnent les fiancés en procession jusqu'à leur nouvelle demeure? La ville, nous dit Chariton, est emplie de chants d'hyménée, les rues de couronnes et de flambeaux, l'entrée des maisons ruisselle de vins et d'onguents (1.1.13). Le resserrement du focus depuis l'ensemble de la ville jusqu'à l'entrée des maisons particulières suggère que la fête est célébrée à tous les niveaux de la communauté civique et qu'elle est prise en charge également à chacun de ces niveaux, civique, vicinal et domestique. Pour ce qui est de la fête dans sa dimension civique, il est probable qu'Hermocrate la prend à sa charge en tant que stratège et premier citoyen, mais cela reste une hypothèse, confortée cependant par l'exemple du prytane Kléanax de Kymè

26 Sur le fait que des descriptions élogieuses précédaient généralement les acclamations décernant l'honneur, cf. Pont 2009, 198-199.

27 Sur les rapports d'ambassade au peuple, $c f$. Fernoux 2011, 269, 271 et 274. 
qui, à l'occasion du mariage de sa fille, offre un banquet public ${ }^{28}$. Le financement des funérailles de Callirhoé est à peine plus explicite. Il n'est pas question de sacrifice ni de banquet, mais seulement d'une procession officielle. Les offrandes funèbres sont constituées, quant à elles, de la dot de Callirhoé, des parts de butin rapportées par Hermocrate (sa part personnelle probablement), des présents des parents et des amis, puis de toute la fortune de Chairéas qu'il destine à un grand holocauste expiatoire (1.6.4). Le lit funèbre fait d'or massif n'ayant pas été évoqué plus tôt, notamment lors de la décision de la cité d'offrir à la jeune fille des funérailles officielles, on peut supposer qu'il a été réalisé aux frais d'Hermocrate. Le financement de l'expédition navale destinée à ramener Callirhoé à Syracuse ne fait l'objet d'aucune indication précise, même lorsque les bouleutes se proposent d'y participer. En effet, les ambassades pouvaient être prises en charge tantôt par les cités, tantôt par des bienfaiteurs ${ }^{29}$. Mais l'affrètement de la « fameuse trière amirale » $(3 \cdot 5 \cdot 2-3)$ semble être pour la cité une manière de prendre à sa charge une part du coût de l'ambassade. En effet, le choix de cette embarcation est destiné, nous dit Chariton, à donner un caractère officiel à l'ambassade et à « renforcer le prestige de la délégation ».

Nous apprenons dans le dernier passage que Chairéas ajoute à l'octroi de la citoyenneté aux trois cents mercenaires le don d'un talent à chacun d'entre eux, ce qui représente une somme absolument considérable ${ }^{30}$, équivalente à 6000 deniers, tandis qu'Hermocrate offre à chaque soldat égyptien présent à Syracuse (et dont le nombre n'est pas stipulé) un lot de terre à cultiver, pris très certainement sur ses propres domaines. Ces deux dons nous informent à tout le moins sur le niveau de richesse qu'un auteur comme Chariton, secrétaire d'un avocat célèbre, imaginait être celui des premiers citoyens d'une grande cité comme Syracuse.

\section{Quelles sont les procédures suivies?}

En ce qui concerne les procédures, le roman de Chariton ne s'éloigne guère du témoignage des inscriptions de la même époque. Le peuple se rassemble en deux lieux essentiellement: le théâtre d'abord, où siège l'Assemblée lors de ses réunions régulières (texte 1), mais aussi lorsqu'elle se réunit de sa propre

\footnotetext{
28 Cf. ci-dessus n. 20.

29 Zuiderhoek 2009, respectivement p. 39 et 76, concernant précisément les ambassades auprès des gouverneurs et des empereurs.

30 Cette somme est à mettre en parallèle avec les distributions en argent étudiées par Zuiderhoek 2009, 100-101, dans une table qui distingue différents types de bénéficiaires.
} 
initiative (textes 3 et 4), l'agora ensuite, où se réunit le tribunal tiré au sort par les magistrats (texte 2). Ces deux lieux sont bien identifiés comme les lieux de réunion principaux de la communauté civique à l'époque impériale :

Généralement lors des réunions du peuple au théâtre deux éléments se conjuguent : un aspect institutionnel, moins rigoureux peut-être dans ses procédures quà l'époque hellénistique, et une attente d'information et de décision en période de crise ou lorsque celle-ci est résolue. Le théâtre est dédié à la communication entre les différentes composantes de la cité ; la simple rumeur suffit à y faire accourir spontanément les foules, pour s'informer, acclamer les bienfaiteurs, réclamer en leur honneur des funérailles publiques ${ }^{31}$.

À côté du théâtre, «centre névralgique de la communauté civique » ${ }^{32}$, l'agora devrait apparaître comme un lieu signifiant une «ouverture de la cité sur l'extérieur », un «lieu où se croisent citoyens et non-citoyens ${ }^{33}$ ». Mais il n'en est rien dans le roman de Chariton (texte 2). Au contraire, l'agora permet de limiter strictement l'auditoire aux magistrats, aux juges et au public composé de tous les citoyens de Syracuse, excluant les femmes et le plèthos qui n'apparaîtront que dans un deuxième temps, au sein du cortège funèbre conduisant Callirhoé au tombeau.

Quant à la procédure généralement décrite par Chariton, elle implique que l'honneur peut être octroyé à la demande de l'honorandus lui-même, à la demande d'un autre citoyen ou bien sur proposition du peuple. Le premier cas de figure est illustré par les funérailles de Callirhoé et par l'envoi d'une ambassade pour la retrouver (textes 2 et 3). Hermocrate fait la proposition auprès du dèmos assistant à l'agora au procès de Chairéas, donc dans un cadre institutionnel détourné de sa fonction première. Après avoir demandé l'acquittement de Chairéas, Hermocrate demande au peuple réuni d'accomplir rapidement les rites funèbres en l'honneur de sa fille (1.5.7). Nous apprenons que cette faveur lui a été accordée lorsque Chariton décrit le luxe des funérailles qui sont alors célébrées (1.6.2), mais une ellipse sépare les deux passages : aucune indication n'est donnée quant à la procédure institutionnelle suivie.

Une comparaison avec les funérailles d'Apollonis nous autorise cependant à apporter deux éléments d'information et à risquer peut-être une hypothèse. D'une part, elle nous permet de mesurer l'importance de l'honneur décerné

33 Pont 2010, 75-77 sur l'agora plèthousa. 
à Hermocrate et à Callirhoé ${ }^{34}$. Même s'il ne semble pas s'agir dans le cas de Callirhoé d'un enterrement intra muros, puisque son tombeau sera l'antique tombeau familial dressé sur une falaise en bord de $\operatorname{mer}^{35}$, en un lieu certainement proche des murailles de la cité mais accessible aux pirates et proche de la plage, l'honneur de funérailles publiques est assez rare et précieux pour être souligné. D'autre part, à la différence des honneurs offerts à Apollonis qui lui sont décernés sur l'initiative du peuple et par acclamation, Callirhoé est honorée sur la proposition de son père. En réalité, le texte étant peu précis à ce sujet, on pourrait comprendre également qu'Hermocrate ait demandé au peuple de ne pas différer plus longtemps par le déroulement de l'enquête le moment d'ensevelir sa fille et que le peuple ait alors proposé d'offrir à la jeune femme des honneurs hors du commun, comme ceux qui pouvaient être décernés aux plus hautes familles de la cité.

Le troisième texte, décrivant l'envoi de l'ambassade, est beaucoup plus circonstancié. Cette fois-ci encore, c'est Hermocrate qui est à l'origine de la faveur qui lui est faite et c'est à l'occasion d'une enquête publique. Le peuple des citoyens de Syracuse est en effet réuni au théâtre, toutes classes confondues, comme l'atteste la présence d'un pêcheur au milieu de l'assistance, de même que leurs épouses - «Même les femmes siégèrent à cette assemblée», écrit

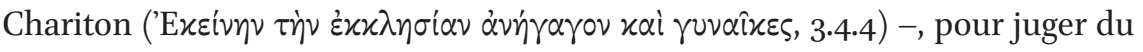
sort du pirate Théron qui a été arrêté en mer alors qu'il voguait à la dérive sur un bateau chargé des nombreuses offrandes déposées dans le tombeau de Callirhoé. Chairéas fait le récit de cette découverte, refusant en signe de deuil de monter à la tribune pour témoigner, ce qui atteste qu'une tribune était dressée au théâtre pour permettre la tenue des débats de l'Ecclésia. Puis Théron est torturé dans le théâtre même où il avoue son forfait (3.4.12), et le peuple vote

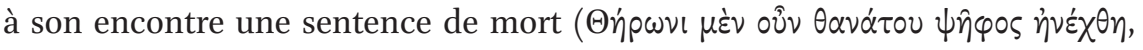
3.4.15). Hermocrate propose alors d'exécuter la sentence à la suite d'un court débat qui l'oppose à Chairéas (qui souhaite, pour sa part, poursuivre l'épreuve de la torture pour obtenir du pirate plus de renseignements), et demande à ses concitoyens qu'ils fassent rechercher sa fille. Il invoque le mérite de sa stratégie et de ses victoires pour susciter la reconnaissance du peuple interpellé au moyen de l'apostrophe habituelle (3.4.16): «Je vous demande, citoyens de Syracuse, de vous rappeler ma stratégie et de me témoigner maintenant, à pro-

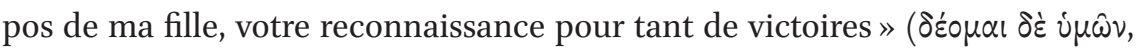

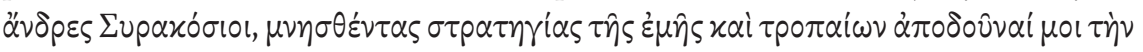

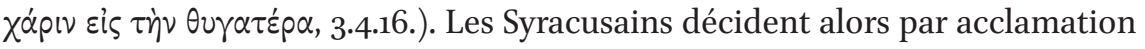

34 Cf. C. Kuhn dans le présent volume.

35 Rife 1999, 96-102.

For use by the Author only | (c) 2017 Koninklijke Brill NV 


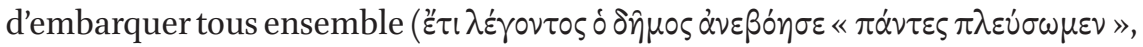
3.4.17). C'est dans un deuxième temps, lorsque presque tous les bouleutes se seront également proposés pour faire partie de l'expédition, qu'Hermocrate définira les règles selon lesquelles sera composée l'ambassade, non sans avoir remercié ses concitoyens pour l'honneur (timè) qui lui a été fait: l'ambassade comportera deux délégués pris parmi le dèmos et deux autres choisis dans la boulè, auxquels s'ajoutera Chairéas. La proposition est ensuite acceptée par le peuple et la décision ratifiée officiellement, probablement par la rédaction

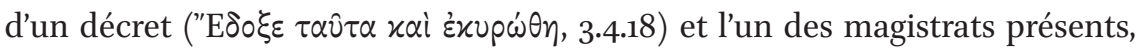
sans doute Hermocrate mais le sujet du verbe n'est pas spécifié, dissout l'as-

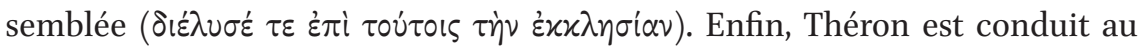
supplice de la crucifixion.

L'Assemblée qui ratifie l'envoi de cette ambassade est en fait la continuation d'une assemblée convoquée par Hermocrate pour mener une enquête officielle sur la disparition du corps de Callirhoé dans son tombeau et pour juger le chef des pirates Théron (3.4.3). La décision d'envoyer une ambassade n'était donc pas inscrite à l'ordre du jour initial, lui-même fixé en toute hâte par Hermocrate, «homme de commandement et d'expérience » (3.4.3), et s'impose spontanément à l'issue de la condamnation de Théron ${ }^{36}$. Que l'Assemblée soit l'organe de décision convenant à l'envoi d'une ambassade est bien attesté ${ }^{37}$.

Dans un seul cas, il arrive qu'un citoyen soit à l'origine des honneurs décernés par le peuple à un autre citoyen. Il s'agit du passage où Chairéas fait la proposition d'honorer Polycharme et les mercenaires grecs qui ont combattu à ses côtés. Il décrit très précisément les honneurs qui pourraient leur être rendus, à Polycharme un éloge public et des noces officielles avec sa propre sœur (honneur dont le caractère romanesque emprunte évidemment son modèle à ses propres noces), et à ses trois cents compagnons d'armes l'octroi collectif de la citoyenneté, honneur dont il faut relever ici la rareté et le caractère exceptionnel, que ce soit à titre individuel ${ }^{38}$ ou collectif ${ }^{39}$. Dans le cas qui nous

36 Sur le pouvoir d'initiative de l'Assemblée quant aux questions qui l'intéressent, $c f$. Fernoux 2011, 191-192.

$C f$. Fernoux 2011, 266-271. C'est en effet une prérogative du peuple qu'à partir du II ${ }^{\mathrm{e}}$ siècle ap. J.-C. un certain nombre de grands notables commencent à s'arroger, et sur laquelle les empereurs finiront par légiférer pour en limiter les coûts.

38 Oliver 2007 .

39 Les rares cas recensés par les sources épigraphiques d'octroi collectif de la citoyenneté concernent des groupes d'individus originaires de cités bénéficiant d'accords d'isopolitie. Cet honneur attribué à des compagnons d'armes au titre de leurs prouesses guerrières est davantage du ressort de la fantaisie romanesque que de la réalité des cités grecques d'époque impériale. 
occupe, la citoyenneté est immédiatement effective, ce que signifie clairement l'accueil des trois cents nouveaux citoyens sur les sièges de l'Assemblée ${ }^{40}$. Elle est entièrement du ressort de la volonté populaire.

Le peuple enfin peut être à l'origine de la proposition d'honorer un citoyen. Le premier texte en est le meilleur exemple. À l'occasion d'une réunion régulière de son assemblée (1.1.11), le dèmos fait lui-même la proposition d'organiser les noces officielles et publiques de Chairéas et Callirhoé dont Hermocrate désapprouve personnellement le projet. De cette assemblée dont Éros est le «meneur», nous dit Chariton ( $\hat{\eta} \varsigma$ o "E rable (1.1.12): «En bon patriote, Hermocrate ne put s'opposer à la démarche

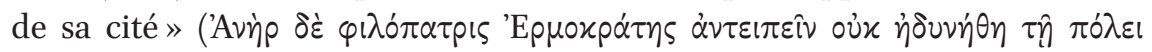
$\delta \varepsilon \circ \mu \varepsilon \dot{v} \eta$ ). Cette fois, c'est Hermocrate qui sanctionne la décision du peuple en donnant son accord et le peuple se précipite hors du théâtre pour organiser les festivités. Tous les cas de figure sont donc possibles, que la proposition émane du peuple ou d'un ou plusieurs citoyens, y compris l'honorandus lui-même. Comme il se doit dans un régime démocratique, tout citoyen peut proposer que soient décernés des honneurs à un individu particulièrement méritant.

Ces honneurs sont généralement décernés par acclamation, ce que traduit dans le roman de Chariton l'utilisation du verbe $\beta 0 \alpha \omega \omega(1.1 .11 ; 3.4 .17 ; 8.7 .1$;

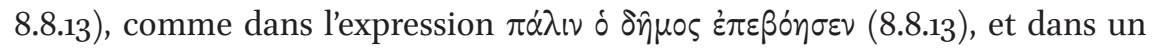

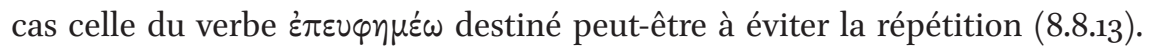
Lacclamation est une manière bien attestée pour le dèmos de décerner des honneurs depuis l'époque hellénistique, qu'il s'agisse de simples citoyens, de grands bienfaiteurs, de gouverneurs ou d'empereurs ${ }^{41}$. Elle permet à Chariton, comme aux cités qui faisaient graver le contenu ou la simple mention de décrets honorifiques, de souligner le caractère d'universalité de cette décision prise par le dèmos dans son ensemble ainsi que la pression exercée par la liesse populaire $^{42}$.

Cependant, à plusieurs reprises, l'acclamation doit être ratifiée et confirmée par un acte officiel émanant des institutions de la cité. Lors du procès de Chairéas d'abord, le peuple présent sur l'agora pour assister aux débats est touché par l'accusation que le jeune homme porte contre lui-même et «[se détourne] de la morte pour pleurer le vivant» (1.5.6). Ce sont cependant les juges, tirés au sort le jour même par les magistrats (1.5.2), qui portent la

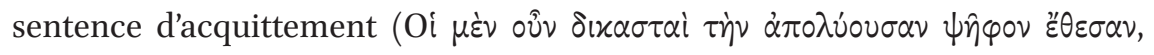

\footnotetext{
40 Dans ce cas précis, la citoyenneté n'est pas seulement potentielle. $C f$. Gauthier 1985, 150, et l'introduction d'A. Heller et A.-V. Pont dans Heller, Pont 2012, part. 11.

41 Pont 2009, 198-202; Fernoux 2011, 134-147.

$42 \quad C f$. C. Kuhn dans le présent volume.
} 
1.6.1 ${ }^{43}$. Dans le cas de l'envoi de l'ambassade sur une décision de l'Ecclèsia réunie au théâtre (texte 3), la décision est ratifiée officiellement (3.4.18) puis l'assemblée dissoute, sans doute par Hermocrate lui-même bien que Chariton

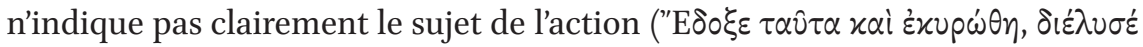

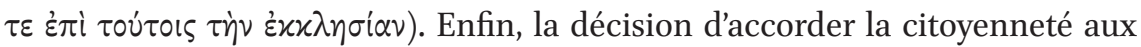
trois cents mercenaires grecs de l'Égyptien qui ont combattu aux côtés de Chairéas est bien prise par le peuple par acclamation, mais cette décision qui

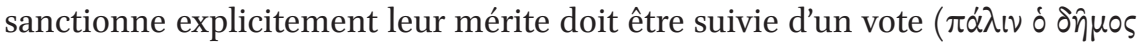

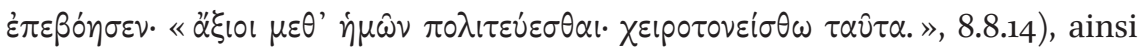

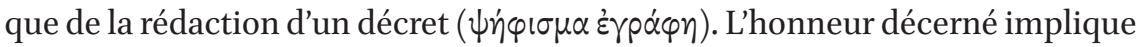
notamment que soit dressée et inscrite une liste de noms ou politographie ${ }^{44}$. S'ensuit l'accueil immédiat des nouveaux citoyens dans les rangs de l'Assem-

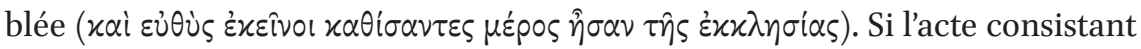
à accorder collectivement la citoyenneté à trois cents Grecs d'origines diverses revêt un caractère tout à fait exceptionnel, relevant de la création romanesque, il suit toutefois une procédure régulière et ne se dispense pas des formalités nécessaires dans ce genre de gratification officielle ${ }^{45}$.

Élément intéressant, au cours de ces procédures d'octroi d'honneurs civiques, Chariton évoque le dèmos et le plèthos comme deux entités séparées ${ }^{46}$. Alors que le premier terme désigne le corps civique de Syracuse, le second recouvre la foule des Syracusains qui se réunit dans la ville, à l'agora, en bord de mer, au port ou dans la nécropole, pour accompagner chaque événement important de la vie privée de la première famille de la cité, celle d'Hermocrate et de Callirhoé. Ainsi, seul le dèmos est réuni en Assemblée régulière au début du roman (texte 1) lorsque se prend la décision de marier les deux jeunes héros (1.1.11); c'est lui également qui assiste au procès de Chairéas à l'agora (1.5.3) et lui très certainement qui compose le tribunal tiré au sort par les magistrats (1.5.2). Dans les deux cas, le plèthos se joint ensuite au dèmos pour participer à la mise en œuvre de la décision prise par le peuple. Si le dèmos accompagne les deux jeunes époux vers leur nouveau domicile (1.1.12-13), plus tard, lorsque Schwartz 1998, chapitre 2, « Chariton : Trials in the Greek City », 39-71.

Nous connaissons un certain nombre de politographies, pour l'époque hellénistique surtout, comme la politographie de Pharsale (IG IX.2, 234) ou celles du Delphinion de Milet et d'Ilion étudiées par Brulé 1990. Cf Savalli 1985.

45 Oliver 2007.

46 Pour une étude de ces notions à l'époque classique, principalement dans la Constitution des Athéniens d'Aristote, $c f$. Ruzé 1984, part. 259-263; Keaney 1992, chapitre 16, « $\Delta \dot{\eta} \mu \circ \varsigma$, $\pi \lambda \hat{\eta} \theta \circ \varsigma$ and $\pi \dot{\lambda} \lambda ı \varsigma », 156-166$; Ober 2001, 332-339; Ober 2009, 11, part. n. 14 et 15. 
le cortège est rompu et que Callirhoé s'avance vers la foule, ce n'est plus le

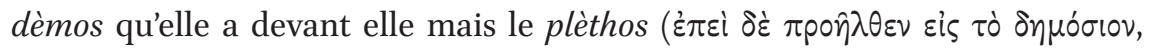

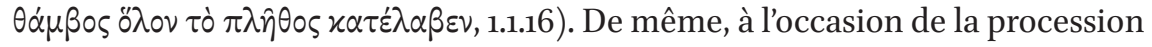
civique qui accompagne ses funérailles (texte 2), c'est la foule des Syracusains

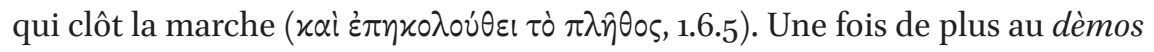
s'adjoint le plèthos, mais dans un deuxième temps, dans un groupe séparé et en seconde place. On voit clairement dans ces deux exemples qu'il s'agit là de deux acteurs différents de la vie publique, le plèthos incluant les épouses de citoyens - étrangers domiciliés à Syracuse, étrangers de passage et esclaves ne sont jamais mentionnés. Précisons que chez Chariton, le terme plèthos ne semble pas revêtir un sens péjoratif comme c'est généralement le cas dans les sources littéraires ${ }^{47}$.

Les troisième et quatrième textes introduisent une nouveauté, la participation des femmes, c'est-à-dire des épouses de citoyens, au rassemblement du peuple, ce qui explique l'emploi du terme plèthos pour désigner la foule qui accompagne le dèmos du bord de la mer au théâtre, puis du théâtre au tombeau de Callirhoé (texte 3). Il est délicat d'établir le statut de ces assemblées spontanées élargies aux femmes de citoyens : attestation d'une réalité gommée par les déclarations officielles que nous ont transmises les inscriptions ou fantaisie romanesque? Lorsque l'Ecclésia est convoquée par Hermocrate pour mener une enquête sur la disparition du corps de Callirhoé (texte 3), celle-ci est réunie au théâtre, ce qui est conforme aux pratiques habituelles, mais les femmes y siègent aussi bien que les hommes, nous dit Chariton ( $\dot{\varepsilon} \varkappa \varepsilon i \nu \eta \nu \nu \dot{\nu} \nu \dot{\varepsilon} \varkappa \varkappa \lambda \eta \sigma^{\prime} \alpha \nu$

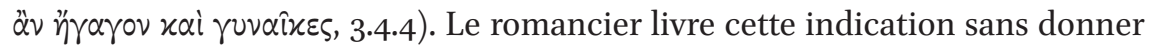
l'impression de s'en étonner, mais le fait même qu'il précise le fait indique son caractère à tout le moins particulier, sinon exceptionnel. Il lui offre ainsi l'occa-

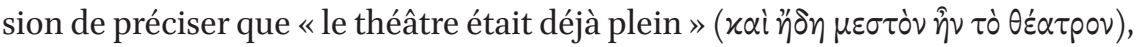
renforçant le sentiment d'une immense sympathie populaire pour le couple des jeunes héros issu des premières familles de la cité. Il faut rappeler que la foule s'était au préalable massée au bord de la mer, devant le tombeau vide (3.4.1), alertée par la rumeur qui se hâte de propager les nouvelles extraordinaires, et que c'est probablement la même foule qui a suivi ensuite le peuple au théâtre. Cependant, la procédure suivie, ainsi que les agents intervenant

H. Fernoux remarque en effet un décalage entre les textes littéraires, qui s'appuient sur une conception élitiste du plèthos comme d'une « masse versatile qu'il importe de mater » et qui est héritée des auteurs du $\mathrm{IV}^{\mathrm{e}}$ siècle av. J.-C., et les documents épigraphiques qui le présentent plutôt comme la «communauté unanime des citoyens ». $C f$. Fernoux 2011, 124-129. Chariton est donc plus proche du langage des inscriptions que de celui d'un Dion de Pruse, par exemple. 
au cours de l'enquête (magistrats, esclaves publics, bouleutes) sont conformes aux réalités de l'époque impériale attestées par les inscriptions ${ }^{48}$, et si Chairéas et le pirate Théron adressent directement à la foule leur récit, c'est bien aux

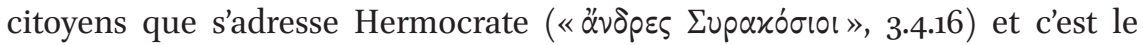

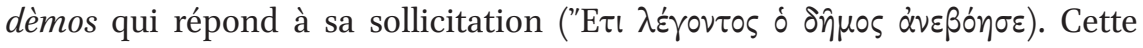
réduction du public au cercle des seuls citoyens pourrait nous conduire à supposer que les femmes de citoyens siègent à l'Assemblée, mais sans avoir le droit

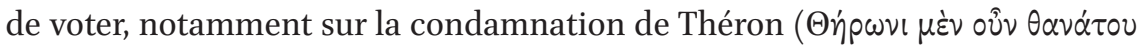

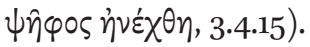

Les femmes participent-elles cependant aux acclamations, et donc à la prise de décision? Il est impossible de le déduire de ce passage, mais le quatrième texte apporte une précision importante à ce sujet, même s'il introduit un certain flottement dans la distinction faite habituellement entre dèmos et plèthos par Chariton. Dans ce texte, en effet, il est précisé qu' « un cri massif monta de

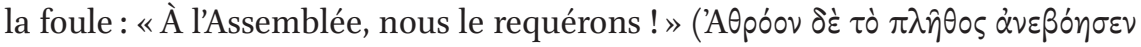

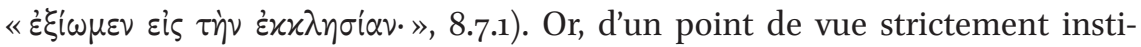
tutionnel, le plèthos ne devrait pouvoir appeler qu'à une réunion au théâtre, et non à l'Assemblée. Mais il arrive alors que les hommes et les femmes rem-

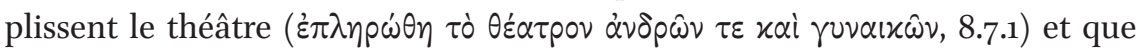
«toutes et tous», dans cet ordre précisément, exigent par acclamation que

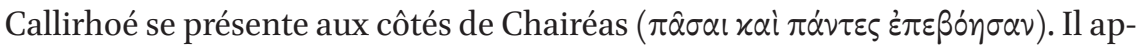
paraît donc manifeste que, dans le monde de Chariton, les femmes libres, sans doute épouses de citoyens, peuvent participer aux acclamations.

Dans la suite du texte décrivant l'octroi de la citoyenneté aux trois cents mercenaires grecs, Chariton livre une précision intéressante. Il nous dit en effet qu'après avoir été honorés de cette faveur que nous savons exceptionnelle, les nouveaux citoyens sont aussitôt admis sur les gradins de l'Assemblée

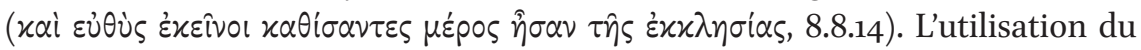

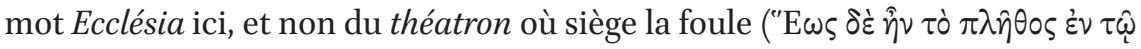
$\theta \varepsilon \alpha \dot{\tau} \rho \omega, 8.8 .15)$, suggère qu'il pourrait y avoir une division hiérarchique de l'espace entre les bouleutes siégeant dans les premiers rangs du théâtre - certains d'entre eux, honorés de la proédrie, trônant sur des sièges sculptés au milieu du tout premier rang, comme en témoignent aujourd'hui encore les vestiges archéologiques des théâtres d'époque hellénistique et romaine -, les citoyens, siégeant ensemble dans la partie basse de l'hémicycle et accueillant parmi eux

48 Pont 2010, 113-114, qui souligne également le fait que «cet épisode décrit des pratiques habituelles en marquant ce qui sort de l'ordinaire : normalement les femmes ne se réunissent pas à l'assemblée ». 
les nouveaux citoyens, et les épouses de citoyens assises plus haut (peut-être avec d'autres catégories sociales composant le plèthos $)^{49}$. Cela expliquerait qu'il soit relativement facile pour un orateur de s'adresser aux seuls citoyens, ou pour les citoyens de s'exprimer indépendamment du plèthos. C'est du moins ce que laisse à penser un passage comme celui où le plèthos se lamente en apprenant la servitude à laquelle a été réduit Chairéas à l'occasion de l'une de

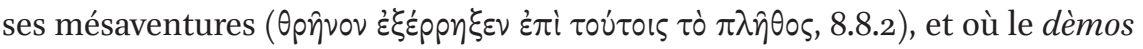

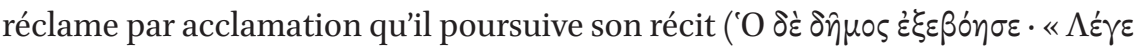
$\pi \dot{\alpha} v \tau \alpha$. ). Ce cloisonnement de l'espace serait assez conforme à ce qui apparaît dans les processions civiques où la notion de hiérarchie des catégories civiques s'affirme avec autant de vigueur que celle de l'unité de la cité.

Il est impossible, en l'état de nos connaissances, d'établir avec certitude que les femmes pouvaient assister, voire participer, à certaines réunions de l'Assemblée. Il reste que, les romanciers s'appuyant toujours sur le vraisemblable pour bâtir leurs intrigues et manifestant généralement des opinions socialement conservatrices, il est improbable que Chariton ait inventé de toutes pièces une situation qui aurait paru scandaleuse à ses lecteurs si elle n'avait pas eu quelque réalité à son époque. On a bien cru pendant longtemps que les femmes n'étaient pas autorisées à assister aux représentations théâtrales données à Athènes au v $\mathrm{v}^{\mathrm{e}}$ siècle av. J.-C., jusqu'à ce que cette affirmation ait été invalidée ${ }^{50}$. Cependant, cette participation féminine pourrait nêtre survenue que dans certains cas particuliers où la foule se réunissait spontanément au théâtre, par exemple ceux où les délibérations impliquaient la famille d'un magistrat, mais aussi dans tous les cas où la communauté civique, femmes comprises, se sentait concernée. Certains textes attestent de manière sporadique, et dans des circonstances particulières, d'une telle participation des femmes à une réunion de l'Ecclèsia au théâtre ${ }^{51}$. Celle-ci devait être possible lorsque

49 Fernoux 2011, 93-95. H. Fernoux précise que les assemblées pouvaient s'ouvrir à d'autres catégories que les citoyens adultes votants et évoque les enfants. Il ne cite cependant pas les femmes.

$50 \quad$ Andrisano 2007.

$51 \quad$ A.-V. Pont relève le fait que de vieilles femmes sont amenées au théâtre dans les Actes apocryphes de Jean datés du $\mathrm{II}^{\mathrm{e}}$ siècle $\left(\right.$ A.Io $\left.3^{3-32}\right)$ et P. Thonemann a étudié, dans un article récent sur lequel $\mathrm{O}$. Van Nijf a bien voulu attirer mon attention, une assemblée de femmes d'Akmoneia en Phrygie réunie autour de 6/7 ap. J.-C. pour accorder des honneurs à une prêtresse du nom de Tatia. Il semble cependant que ce type d'assemblée féminine soit resté un cas isolé. $C f$. Thoneman 2010. 
l'assemblée était large et spontanée ${ }^{52}$, puisque nos sources distinguent ces assemblées des assemblées légales ${ }^{53}$.

Enfin, pour en terminer avec les procédures suivies, et comme en attestent les décrets que nous ont conservés les inscriptions d'époque hellénistique et impériale, toute décision validée pouvait être suivie d'une ou plusieurs dispositions particulières. Nous en avons quelques exemples dans le roman de Chariton. Ainsi, quelque temps après qu'il a été décidé par le dèmos d'envoyer une ambassade à la recherche de Callirhoé (texte 3), sont choisis le moment adéquat pour embarquer, en plein hiver finalement, contre toute règle de prudence mais à la demande pressante de Chairéas (3.5.1), et le navire qui convoiera l'ambassade de la cité, c'est-à-dire la trière amirale (3.5.3) qui a vu la victoire d'Hermocrate sur les Athéniens et qui revêt depuis lors un caractère officiel, et peut-être sacré, équivalent à la Paralienne ou à la Salaminienne à Athènes ${ }^{54}$. À la toute fin du roman également (texte 4), l'insigne honneur décerné par le peuple aux trois cents mercenaires grecs, à savoir l'octroi de la citoyenneté syracusaine, est complété par des récompenses d'ordre privé relevant de la générosité de Chairéas et d'Hermocrate : le premier offre un talent à chacun des trois cents nouveaux citoyens, tandis que le second attribue un lot de terre à chacun des soldats égyptiens qui ont accompagné son gendre en Sicile et l'ont accompagné jusqu'à la victoire. Cette procédure rappelle que des dispositions particulières, relevant du contrôle de la cité ou de la générosité privée, pouvaient suivre l'énoncé de la décision elle-même.

52 Pour d'autres exemples de ce type, $c f$. Pont 2010, 112-114, qui plaide d'ailleurs également en faveur d'une vraisemblance du roman de Chariton : «La cohérence interne du roman recommande que le récit paraisse vraisemblable; aussi les récits de ces trois réunions du peuple au théâtre de Syracuse, qui scandent le roman (à l'ouverture, avant le départ de Chéréas, à la fin) doivent-ils paraître réalistes au lecteur de la fin du $\mathrm{I}^{\mathrm{er}} \mathrm{s}$. ou du début du ${ }{ }^{\mathrm{e}} \mathrm{s}$., sans quoi le roman perd son intérêt. »

$53 C f$. le témoignage de Chariton sur une nomimos Ecclèsia (1.1.11), expression en vigueur à l'époque impériale. $C f$. Pont 2010, 113. On peut citer également le récit rapportant la séance de l'assemblée du peuple d'Éphèse se réunissant pour comprendre l'émeute des orfèvres contre Paul de Tarse (Actes 19.23-40). Le greffier de l'Assemblée rappelle aux citoyens que s'ils souhaitent un procès, ils doivent s'adresser aux proconsuls, et que s'il est question d'autres affaires, il est possible de réunir une assemblée légale, ce que n’est pas l'assemblée en cours.

54 Notons que c'est précisément la Salaminienne qui fut envoyée par les Athéniens pour arrêter Alcibiade alors qu'il commandait l'expédition de Sicile. Il s'agit là d'un clin d'œil historique de Chariton destiné aux lecteurs attentifs. 
Il ressort du témoignage de Chariton d'Aphrodisias que la décision d'honorer un citoyen ou un étranger est toujours prise par le dèmos, réuni au théâtre ou éventuellement à l'agora, et qu'elle est adoptée par acclamation. Ce témoignage met donc une fois de plus en évidence le rôle du dèmos dans la cité grecque d'époque impériale. La proposition d'honorer un citoyen peut être faite par un autre citoyen, par l'honorandus lui-même ou par le peuple. Les assemblées peuvent être régulières ou spontanées. Dans ce dernier cas, elles rassemblent l'ensemble du plèthos et les épouses des citoyens (peut-être toutes les femmes libres) peuvent y assister, voire y participer par leurs acclamations. Il est possible qu'alors une certaine disposition de la foule sur les gradins du théâtre, éloignant progressivement de la tribune ceux dont le statut civique est en quelque sorte moins élevé, permette de distinguer les magistrats et les bouleutes, puis les hommes formant le peuple des citoyens, sans considération de classe toutefois, puis les épouses de citoyens. Dans certains cas, comme l'envoi d'une ambassade ou l'octroi de la citoyenneté, la décision est suivie d'un acte permettant de lui donner force de loi, comme un vote ou l'adoption d'un décret. Dans la totalité de ces textes, et contrairement à l'impression générale qui se dégage du roman et qui fait la part belle aux premières familles de la cité, on observe un certain équilibre des pouvoirs entre d'un côté Hermocrate, Callirhoé et Chairéas, représentants les plus illustres de la classe des notables, et de l'autre le peuple, qui conserve toutes ses prérogatives dans un régime que Chariton présente volontiers comme démocratique. Lélément le plus nouveau est l'implication de la cité aux différentes étapes de la vie familiale des élites ou, pour le dire autrement, la publicité donnée par les notables à certains événements de leur vie privée, essentiellement ceux qui mettent en jeu leur descendance. Ces mêmes événements sont l'occasion d'une implication des épouses de citoyens qui prennent alors toute leur place dans une communauté civique élargie. Il faut rappeler à ce sujet que Callirhoé est la fille unique d'Hermocrate et que l'attention que lui porte cette «cité élargie» dépend en grande partie de son statut particulier dans la filiation de l'illustre stratège, issu de l'une des familles les plus nobles de la cité. Après tout, Chariton comptant lui-même parmi les notables de sa cité d'Aphrodisias, mais, en tant que «secrétaire de rhéteur », dans une sorte de deuxième cercle constitué des petits notables et de ceux qui fréquentent le cercle des grands notables, c'est un point de vue assez original sur la vie civique que son roman nous délivre, un point de vue qui n'est peut-être pas exempt d'une part d'idéalisation, mais qui atteste d'un mode de pensée qui mérite d'être étudié plus avant. 
Annexe 5.1 : Les honneurs civiques dans le roman de Chariton

\begin{tabular}{llll}
\hline Référence & Honorandus & $\begin{array}{l}\text { Institution décernant les } \\
\text { honneurs }\end{array}$ & Procédure d'octroi \\
\hline Texte 1 & Hermocrate, & - le dèmos de Syracuse & - demande émanant du peuple \\
$\mathbf{1 . 1 . 1 1 - 1 3}$ & stratège de & réuni en Ecclèsia & par acclamation \\
& Syracuse & $\begin{array}{l}\text { régulière } \\
\text { - lieu de réunion: }\end{array}$ & - accord du stratège Hermocrate \\
& & le théâtre &
\end{tabular}

\begin{tabular}{|c|c|c|c|}
\hline Texte 2 & Callirhoé et & - le dèmos de Syracuse & - proposition d'Hermocrate auprès \\
\hline $1.5 .2-1.6 .5$ & Hermocrate & $\begin{array}{l}\text { - lieu de réunion: } \\
\text { l'agora }\end{array}$ & $\begin{array}{l}\text { du dèmos assistant au } \\
\text { procès de Chairéas } \\
\text { - les juges (tirés au sort par les } \\
\text { magistrats) acquittent Chairéas } \\
\text { à la demande d'Hermocrate } \\
\text { - le peuple (pantes) se lamente } \\
\text { - Hermocrate obtient } \\
\text { d'accomplir rapidement les rites } \\
\text { funèbres }\end{array}$ \\
\hline Texte 3 & Hermocrate & - le dèmos convoqué & - condamnation à mort du pirate \\
\hline \multirow[t]{12}{*}{$3 \cdot 4 \cdot 3-3 \cdot 5 \cdot 3$} & & par Hermocrate à une & Théron \\
\hline & & assemblée en vue & - proposition d'Hermocrate \\
\hline & & d'une enquête & d'exécuter la sentence et de faire \\
\hline & & officielle sur la & rechercher sa fille (contre la \\
\hline & & mort de sa fille & proposition de Chairéas) \\
\hline & & Callirhoé & - Hermocrate invoque le mérite de \\
\hline & & - lieu de réunion : le & sa stratégie et de ses victoires pour \\
\hline & & théâtre & susciter la reconnaissance du \\
\hline & & - les femmes siègent & peuple \\
\hline & & également au théâtre & - décision par acclamation du \\
\hline & & & peuple de Syracuse d'envoyer une \\
\hline & & & ambassade \\
\hline
\end{tabular}




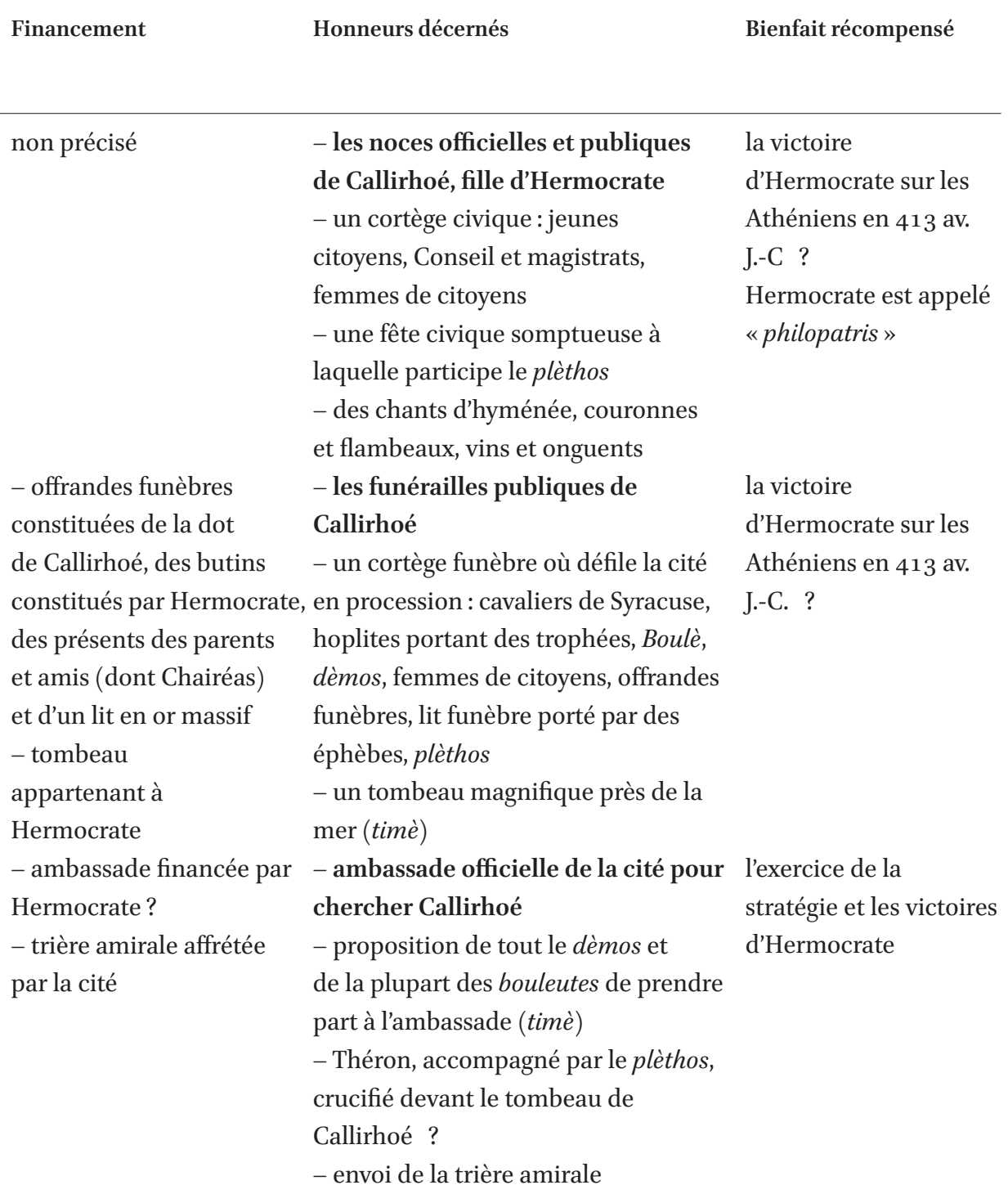


(cont.)

\begin{tabular}{lll}
\hline Référence Honorandus & $\begin{array}{l}\text { Institution décernant les } \\
\text { honneurs }\end{array}$ & Procédure d'octroi \\
& - participation du & - proposition d'Hermocrate sur la \\
plèthos, qui suit le & constitution de l'ambassade : deux \\
dèmos du bord de mer & délégués pris parmi le dèmos, deux \\
au théâtre, puis au & dans la Boulè, et Chairéas \\
tombeau de Callirhoé & - ratification officielle de la \\
& décision par l'Ecclèsia \\
& - dissolution de l'Ecclèsia \\
& - dispositions particulières (saison, \\
& trière amirale de Syracuse)
\end{tabular}

Texte 4 - Chairéas - le dèmos réuni sur sa - toutes et tous convoquent

8.7.1-8.8.14 et Callirhoé propre initiative et par Chairéas et Callirhoé par acclamation pour acclamation

former une Ecclèsia - le dèmos rend grâce aux dieux

- lieu de réunion: le - acclamation de Chairéas par les théâtre hommes et de Callirhoé par les

- les femmes siègent femmes, de tous deux par la foule

également au théâtre entière

- le dèmos demande à Chairéas de faire le rapport de son ambassade - le dèmos adresse aux dieux de nouvelles prières

- Polycharme - le dèmos

- lieu de réunion:

le théâtre

- les trois - le dèmos réuni au

cents théâtre

mercenaires

grecs
- proposition de Chairéas

- sanction du peuple par

acclamation

- proposition de Chairéas

- sanction favorable du peuple par acclamation

- rédaction d'un décret

- accueil immédiat des nouveaux

citoyens dans les rangs de

l'Assemblée

- disposition particulière : don

d'un lot de terre à chaque soldat

égyptien 
- éloges de Chairéas et Callirhoé

- rapport public d'ambassade de Chairéas qui tourne à l'autocélébration

- éloge public du dèmos - noces officielles et publiques avec la sour de Chairéas

- octroi collectif de la citoyenneté syracusaine
- ambassade

couronnée de succès - exploits militaires : prise de Tyr ; victoire navale sur le Grand Roi ; prise d'un butin plus considérable que le trésor des Athéniens ; acquisition de nouveaux alliés

- « des services dignes d'Hermocrate et de Chairéas » - combat aux côtés de Chairéas victorieux talent à chacun

- don d'Hermocrate

\section{For use by the Author only | (C 2017 Koninklijke Brill NV}




\section{Bibliographie}

Andrisano A.M., «Le public féminin du théâtre grec. À propos de la Lysistrata d'Aristophane», Methodos 7, 2007, mis en ligne le 10 avril 2007, consulté le 23 novembre 2014. URL : http://methodos.revues.org/587; DoI : 10.400o/methodos.587.

Baslez M.-F., 1992, «De l'histoire au roman : la Perse de Chariton», dans M.-F. Baslez, P. Hoffmann, M. Trédé (dir.), Le Monde du roman grec, Paris, 199-212.

Bowie E.L., 2002, «The chronology of the earlier Greek novels since B.E. Perry: revisions and precisions », Ancient narrative 2, 47-63.

Bremen van R., 1996, The Limits of Participation. Women and Civic Life in the Greek East in the Hellenistic and Roman Periods, Amsterdam.

Brulé P., 1990, «Enquête démographique sur la famille grecque antique. Etude de liste de politographie d'Asie mineure d'époque hellénistique (Milet et Ilion) », Revue des Etudes Anciennes, 92.3-4, 233-258.

Chankowski A., 2010, L’éphébie hellénistique, Paris.

Ehrhardt N., 2008, «Der Bürger als Heros: Staatsbegräbnis und Öffentlichkeit in Kyzikos », dans E. Winter (ed.), Vom Euphrat bis zum Bosporus : Festschrift für Elmar Schwertheim zum 65. Geburtstag, Bonn, 187-196.

Fernoux H., 2011, Le Dèmos et la Cité. Communautés et assemblées populaires en Asie Mineure à l'époque impériale, Rennes.

Finley M.I., 1968, La Sicile antique. Des origines à l'époque byzantine, trad. J. Carlier, Paris (rééd. 1986).

Gauthier P., 1985, Les Cités grecques et leurs bienfaiteurs, Athènes / Paris.

Heller A., Pont A.-V., 2012, Patrie d'origine et patries électives : les citoyennetés multiples dans le monde grec d'époque romaine, Bordeaux.

Hernandez L.C., 1994, Estudios sobre el aticismo de Cariton de Afrodisias, Amsterdam.

Hodot R., 1982, «Décret de Kymè en l'honneur du Prytane Kléanax », The J. Paul Getty Museum Journal 10, 165-180.

Jones C.P., 1992, «La personnalité de Chariton», dans M.-F. Baslez, P. Hoffmann, M. Trédé (dir.), Le Monde du roman grec, Paris, 161-167.

Karabélias E ., 1990, «Le roman de Chariton d'Aphrodisias et le droit. Renversements de situation et exploitation des ambiguïtés juridiques », dans G. Nenci, G. Thür (dir.), Symposion 1988 : Vortäge zur griechischen und hellenistischen Rechtsgeschichte, Cologne, 369-396.

Keaney J.J., 1992, The Composition of Aristotle's Athenaion Politeia: Observation and Explanation, New York / Oxford.

Lalanne S., 2006, Une éducation grecque. Rites de passage et construction des genres dans le roman grec ancien, Paris.

Lebdiri D., 2015, La religion dans le roman grec ancien, thèse de doctorat, Université Paris 1 Panthéon-Sorbonne. 
Ober J., 2001, Political Dissent in Democratic Athens: Intellectual Critics of Popular Rule, Princeton.

Ober J., 2009, Mass and Elite in Democratic Athens, Princeton.

Oliver Gr., 2007, « Citizenship : Inscribed honours for individuals in classical and hellenistic Athens », dans J.-C. Couvenhes et S. Milanezi (dir.), Individus, groupes et politique à Athènes de Solon à Mithridate, Tours, 273-292.

Oudot E., 1992, «Images d'Athènes dans les romans grecs», dans M.-F. Baslez, P. Hoffmann, M. Trédé (dir.), Le Monde du roman grec, Paris, 101-111.

Pont A.-V., 2009, «Rituels civiques (apantèsis et acclamations) et gouverneurs à l'époque romaine en Asie Mineure » dans O. Hekster, S. Schmidt-Hofner, C. Witschel (dir.), Ritual Dynamics and Religious Change in the Roman Empire, Leyde / Boston.

Pont A.-V., 2010, Orner la cité. Enjeux culturels et politiques du paysage urbain dans l'Asie Mineure gréco-romaine, Bordeaux.

Reardon B.P., 1969, « The Greek novel», Phoenix 23.3, 291-309.

Rife J.L., 1999, Death, Ritual and Memory in Greek Society during the Early and Middle Roman Empire, thèse de doctorat, université du Michigan, Ann Arbor.

Robert L., 1965, Hellenica XIII, Paris.

Robinson E., 2000, «Democracy in Syracuse, 466-412 B.C.», Harvard Studies in Classical Philology, 100, 189-205.

Robinson E.W., 2011, Democracy beyond Athens : popular government in classical Greece, Cambridge.

Rougemont G., 1992, «Delphes chez Héliodore», dans M.-F. Baslez, P. Hoffmann, M. Trédé (dir.), Le Monde du roman grec, Paris, 93-99.

Rutter N.K., 2000, «Syracusan democracy: "Most like the Athenian" »?, in Alternatives to Athens: varieties of political organization and community in ancient Greece, R. Brock and S. Hodkinson ed., Oxford, 137-151.

Ruzé F., 1984, «Plethos. Aux origines de la majorité politique», dans Aux origines de l'hellénisme. La Grèce et la Crète. Mélanges Henrivan Effenterre, Paris, 247-263.

Savalli I., 1985, «I neocittadini nelle città ellenistiche », Historia 34.4, 387-431.

Schwartz S.C., 1998, Courtroom Scenes in the Ancient Greek Novels, thèse de doctorat, université de Columbia, New York.

Schwertheim E., 1978, « Ein postumer Ehrenbeschluss für Apollonis in Kyzikos », ZPE 29, $213-228$.

Sève M., 1979, « Un décret de consolation à Cyzique », BCH 103.1, 327-359.

Thonemann P., 2010, « The women of Akmoneia », JRS 100, 163-178.

Tilg S., 2010, Chariton of Aphrodisias and the Invention of the Greek Love Novel, New York.

Vatin C., 2004, Ariane et Dionysos. Un mythe de l'amour conjugal, Paris. 